\title{
Inclination of magnetic fields and flows in sunspot penumbrae
}

\author{
K. Langhans ${ }^{1}$, G. B. Scharmer ${ }^{1}$, D. Kiselman ${ }^{1}$, M. G. Löfdahl ${ }^{1}$, and T. E. Berger ${ }^{2}$ \\ 1 The Institute for Solar Physics of the Royal Swedish Academy of Sciences, AlbaNova University Center, 10691 Stockholm, \\ Sweden \\ e-mail: [kai; scharmer;dan;mats] @astro.su.se \\ ${ }^{2}$ Lockheed Martin Solar and Astrophysics Lab, 3251 Hanover St., Palo Alto, CA 94304, USA \\ e-mail: berger@lmsal.com
}

Received 11 January 2005 / Accepted 22 February 2005

\begin{abstract}
An observational study of the inclination of magnetic fields and flows in sunspot penumbrae at a spatial resolution of 0.2 is presented. The analysis is based on longitudinal magnetograms and Dopplergrams obtained with the Swedish 1-m Solar Telescope on La Palma using the Lockheed Solar Optical Universal Polarimeter birefringent filter. Data from two sunspots observed at several heliocentric angles between $12^{\circ}$ and $39^{\circ}$ were analyzed. We find that the magnetic field at the level of the formation of the Fe I-line wing $(630.25 \mathrm{~nm})$ is in the form of coherent structures that extend radially over nearly the entire penumbra giving the impression of vertical sheet-like structures. The inclination of the field varies up to $45^{\circ}$ over azimuthal distances close to the resolution limit of the magnetograms. Dark penumbral cores, and their extensions into the outer penumbra, are prominent features associated with the more horizontal component of the magnetic field. The inclination of this dark penumbral component - designated B - increases outwards from approximately $40^{\circ}$ in the inner penumbra such that the field lines are nearly horizontal or even return to the solar surface already in the middle penumbra. The bright component of filaments - designated A - is associated with the more vertical component of the magnetic field and has an inclination with respect to the normal of about $35^{\circ}$ in the inner penumbra, increasing to about $60^{\circ}$ towards the outer boundary. The magnetogram signal is lower in the dark component $\mathrm{B}$ regions than in the bright component A regions of the penumbral filaments. The measured rapid azimuthal variation of the magnetogram signal is interpreted as being caused by combined fluctuations of inclination and magnetic field strength. The Dopplergrams show that the velocity field associated with penumbral component B is roughly aligned with the magnetic field while component A flows are more horizontal than the magnetic field. The observations give general support to fluted and uncombed models of the penumbra. The long-lived nature of the dark-cored filaments makes it difficult to interpret these as evidence for convective exchange of flux tubes. Our observations are in broad agreement with the two component model of Bellot Rubio et al. (2003), but do not rule out the embedded flux tube model of Solanki \& Montavon (1993).
\end{abstract}

Key words. Sun: magnetic fields - Sun: photosphere - Sun: sunspots

\section{Introduction}

Sunspot penumbrae, as seen in continuum images, have traditionally been described as consisting of alternately bright and dark narrow filaments that extend radially from the umbra to the photosphere. The origin of this filamentary structure, and the associated Evershed flow, is not clear, although several mechanisms have been proposed (convection rolls, Danielson 1961a,b; siphon flow, e.g. Thomas 1988; Degenhardt 1989, 1991; convective exchange of flux tubes, Spruit 1981; Jahn 1992; Schlichenmaier et al. 1998a,b; convective pumping of magnetic flux, Weiss et al. 2004). In addition, direct observations of convection within or below the visible layers in the penumbra needed to supply the radiated energy is missing.

In order to establish observational evidence to support or refute existing theories related to penumbral fine structure, substantial efforts have been made to obtain information about penumbral magnetic fields and flow fields at the highest possible spatial resolution. Beckers \& Schröter (1969) were the first to describe the azimuthal inhomogeneity of penumbral magnetic fields. These conclusions were confirmed by Title et al. (1993) (see also Title et al. 1992), who introduced the concept of a fluted penumbra to describe this filamentary configuration of the magnetic field. Their study was based on longitudinal magnetogram data, obtained at the Swedish Vacuum Solar Telescope, with a spatial resolution of about 0.'5. They interpreted the azimuthal fluctuations as being caused by fluctuations of the inclination of the vector magnetic field at the scale of penumbral filaments of about $\pm 18^{\circ}$ about the mean. Using Stokes $V$ data, Schmidt et al. (1992) and Rimmele (1995) found similar fluctuations of the penumbral magnetic field, but, as in Title et al. (1993), no fluctuations of the field strength. Degenhardt \& Wiehr (1991), also using Stokes $V$ only, found a first indication that the more vertical component of the 
magnetic field is related to larger field strength than the more horizontal component. Lites et al. (1993) called these spokelike extensions of the more vertical orientated magnetic field spines. Their studies, based on full Stokes data, show that the inclination fluctuations are highly correlated with field strength fluctuations in the sense that the magnetic field of spines is stronger than in the region between spines, which they called intra-spines (cf. Stanchfield et al. 1997; Westendorp Plaza et al. 1997; Westendorp Plaza et al. 2001; Mathew et al. 2003). Finally, Martinez Pillet (1997) demonstrated that a combination of field strength and inclination variation can explain the azimuthal variations observed by Title et al. (1993).

The distribution of the Evershed flow over the penumbra also shows a filamentary structure (e.g. Johannesson 1993; Shine et al. 1994; Rimmele 1995; Hirzberger \& Kneer 2001), indicating that the bulk of the outflow is concentrated in certain filaments, the Evershed channels. While the existence of an azimuthal variation of the penumbral flow field is well established, conflicting interpretations exist concerning the relationship between the Evershed flow and the observed brightness variations. Beckers (1968) reported that the Evershed flow is related to dark filaments (confirmed by e.g. Title et al. 1993; Rimmele 1995; Stanchfield et al. 1997; Rouppe van der Voort 2002; Tritschler et al. 2004), while the opposite was observed by e.g. Wiehr \& Stellmacher (1989) and Lites et al. (1990).

The existence of a correlation between the velocity field and the magnetic field appears well established. The Evershed flow is mainly concentrated in the inter-spines where the field is weaker and more horizontal (e.g. Skumanich \& Lites 1994; Stanchfield et al. 1997). However, the magnetic field vector seems to be less inclined than the velocity vector by as much as $10^{\circ}$ (e.g. Adam \& Petford 1991). This implies that material motions do not occur along the lines of magnetic force. In their analysis, Bellot Rubio et al. (2003), partly compensates for the limited spatial resolution in the observed data by allowing two magnetic atmospheres to coexist in one resolution element. The analysis indicates two distinct components, one of which has a much more horizontal magnetic field than the other, the inclination of which furthermore appears to be parallel to the flow field. This is consistent with the presence of inclined flux tubes embedded in a more vertical background field, as proposed by Solanki \& Montavon (1993) in their uncombed penumbra model. Lites et al. (2002) presented a twocomponent analysis of a $\delta$-configuration sunspot that results in a fluted magnetic field geometry. Using the MISMA framework (MIcro-Structured Magnetic Atmospheres) Sánchez Almeida (2005b,a) presents a scenario for the sunspot magnetic field, in which short narrow magnetic loops fill the penumbral volume. The scenario explains the small-scale structure of the penumbra in consistence with most of the existing observations.

In spite of this progress an improvement in the spatial resolution of observed data is clearly needed to establish in a more direct way the fine structure of the penumbra. Recently, observations using the Swedish 1-m Solar Telescope (SST, Scharmer et al. 2003a) that achieved a spatial resolution of 0.1 have demonstrated unexpected fine structure in penumbral filaments (Scharmer et al. 2002). The observations show penumbral filaments that consist of unresolved dark cores (measured widths of $90 \mathrm{~km}$ ), flanked by lateral brightenings. These observations are exciting because they suggest that we may be resolving the fundamental physical scales of penumbrae and that further observational diagnostics of the dark cores and their relation to the surrounding bright components of the filaments will give important clues to the understanding of penumbrae. Such observations, based on broadband observations have been made by Rouppe van der Voort et al. (2004) and Sütterlin et al. (2004) but because of the lack of magnetogram, Dopplergram or spectro-polarimetric data at comparable high spatial resolution, the organization of the penumbral magnetic and the flow field remains at these small scales unknown.

In this paper, we present an analysis based on highresolution magnetograms and Dopplergrams using a purely geometrical method (cf. Kinman 1952), e.g. applied by Title et al. (1993) on magnetograms and Schlichenmaier \& Schmidt (2000) on Doppler maps of sunspots. In our data of high spatial resolution we see that the penumbra is highly structured both in continuum filtergrams and magnetograms. We use these azimuthal fluctuation in the measured signal to separate the penumbra spatially in two components and define binary masks. In this way, properties of each visible component can be analyzed separately. The paper is organized as follows: Sect. 2 gives information about the observations; in Sect. 3 we describe the data reduction and analysis; the results are presented in Sect. 4; finally, Sect. 5 summarizes and discusses our findings.

\section{Observations}

All observations were acquired with the SST at the Observatorio del Roque de los Muchachos (La Palma, Spain) (Scharmer et al. 2003a) using the integrated adaptive optics system (Scharmer et al. 2003b). The observations include an isolated small symmetric sunspot of the active region NOAA 10425, observed on three different days: August 4, 2003 $(\mu=0.76)$, August 8, $2003(\mu=0.97)$ and August 9, 2003 $(\mu=0.88)$ and a large less symmetric sunspot (NOAA 10397), observed on three different days: July 1, $2003(\mu=0.85)$, July 2, 2003 ( $\mu=0.98)$, July 5, 2003 ( $\mu=0.90)$.

Filtergrams in circular polarized light in the blue wing of Fe I $630.2 \mathrm{~nm}-5 \mathrm{pm}$ from line center - were recorded with the Lockheed Solar Optical Universal Polarimeter (SOUP) tunable birefringent filter (7.2 pm FWHM at $630.2 \mathrm{~nm}$, cf. Berger \& Title 2001) preceded by a left circular polarization $(L C P)$ filter, a right circular polarization $(R C P)$ filter as well as a blocking filter. Images through the SOUP filter were obtained with an integration time of $130 \mathrm{~ms}$ (August 9, $140 \mathrm{~ms}$ ). The plate scale was $0 .{ }^{\prime} 075 /$ pixel.

Broadband filtergram images were also taken with each pair of SOUP filter images. These were recorded through a $1.15 \mathrm{~nm}$ wide interference filter centered at $436.39 \mathrm{~nm}$ with an integration time of $18 \mathrm{~ms}$ and a plate scale of $0.041 /$ pixel. These images were used as reference images in the data analysis. True continuum images obtained with the SOUP filter tuned $35 \mathrm{pm}$ from the Fe I $630.25 \mathrm{~nm}$ were also recorded and used to align the $L C P$ and $R C P$ magnetogram images to the broadband filtergram images. 
Table 1. Data sets: REF - Reference image, MB - magnetogram in the blue wing of Fe I 630.2 nm, DG - Dopplergram. For the August data REF refers to broadband continuum images, taken at $436.39 \mathrm{~nm}$. For the July data REF refers to narrow-band continuum images, obtained with the SOUP at $-35 \mathrm{pm}$ distance from the line-core of Fe I.

\begin{tabular}{|c|c|c|c|c|}
\hline Date & Time & $\mu$ & $\vartheta\left[^{\circ}\right]$ & Type of data \\
\hline \multicolumn{5}{|c|}{ Small symmetric sunspot of NOAA 10425 : } \\
\hline \multirow[t]{3}{*}{ August 4} & $11: 37$ & 0.766 & 40.0 & 2 pairs: $\mathrm{REF}+\mathrm{MB}$ \\
\hline & $12: 42$ & 0.777 & 39.0 & 6 pairs: $\mathrm{REF}+\mathrm{MB}$ \\
\hline & $14: 25$ & 0.788 & 38.0 & 2 pairs: $\mathrm{REF}+\mathrm{MB}+\mathrm{DG}$ \\
\hline \multirow[t]{2}{*}{ August 8} & 10:02 & 0.962 & 15.8 & 2 pairs: $\mathrm{REF}+\mathrm{MB}$ \\
\hline & $10: 42$ & 0.962 & 15.8 & 1 pair: $\mathrm{REF}+\mathrm{MB}$ \\
\hline \multirow[t]{2}{*}{ August 9} & $11: 45$ & 0.883 & 28.0 & 2 pairs: $\mathrm{REF}+\mathrm{MB}$ \\
\hline & & 0.883 & 28.0 & 2 pairs: $\mathrm{REF}+\mathrm{MB}+\mathrm{DG}$ \\
\hline
\end{tabular}

Large less symmetric sunspot of NOAA 10397:

\begin{tabular}{lcccl} 
July 1 & $12: 44$ & 0.884 & 27.9 & 2 pairs: $\mathrm{MB}$ \\
July 2 & $12: 52$ & 0.967 & 14.8 & 1 pair: $\mathrm{REF}+\mathrm{MB}$ \\
& $14: 58$ & 0.975 & 12.8 & 1 pair: $\mathrm{MB}$ \\
& $16: 29$ & 0.984 & 10.3 & 1 pair: $\mathrm{MB}$ \\
July 5 & $9: 45$ & 0.897 & 26.2 & 3 pairs: $\mathrm{REF}+\mathrm{MB}+\mathrm{DG}$ \\
& $11: 55$ & 0.889 & 27.2 & 2 pairs: $\mathrm{REF}+\mathrm{MB}+\mathrm{DG}$ \\
\hline
\end{tabular}

All images were obtained in frame selection mode. For the broad band channel the best three frames within $12 \mathrm{~s}$ were selected, for the SOUP images the best three images during five seconds were selected for each wavelength and/or polarization state. These three frames were post-processed using Multi Frame Blind Deconvolution (MFBD; Löfdahl 2003). A complete data pair consists of one continuum image as reference and one magnetogram, where all individual frames were obtained within $12 \mathrm{~s}$. For a data pair that includes a Dopplergram all frames on which the pair is based on were obtained within $24 \mathrm{~s}$. All data sets included in the analysis are listed in Table 1 . The common field-of-view is $74^{\prime \prime} \times 74^{\prime \prime}$.

\section{Data reduction and analysis}

\subsection{Data calibration}

After gain and dark correction the spectral filtergrams were restored using the MFBD algorithm of Löfdahl (2003). Simulations show that MFBD applied to long-exposure data ( $>100 \mathrm{~ms}$ ) corrects mainly for the modulation transfer function of the telescope. MFBD co-adds the frames with a 10" resolution correction for anisoplanatic deformation and filters the restored image from noise. For short-exposure data $(\simeq 10 \mathrm{~ms})$, MFBD also corrects for low-order wavefront aberrations. See the appendix in Berger et al. (2004) for details. The SOUP continuum images were carefully scaled and aligned (using bicubic interpolation) to the broadband filtergram images in order to establish the scale transformation parameters. We computed magnetograms, $M^{\vartheta}$, and Dopplergrams, $V^{\vartheta}$, in the following manner:

$$
\begin{aligned}
& M^{\vartheta}=\frac{I_{\mathrm{LCP}}^{\text {blue }}-I_{\mathrm{RCP}}^{\text {blue }}}{I_{\mathrm{LCP}}^{\text {blue }}+I_{\mathrm{RCP}}^{\text {blue }}} \\
& V^{\vartheta}=f_{\mathrm{c}} \frac{\left(I_{\mathrm{LCP}}^{\text {blue }}+I_{\mathrm{RCP}}^{\text {blue }}\right)-\left(I_{\mathrm{LCP}}^{\text {red }}+I_{\mathrm{RCP}}^{\text {red }}\right)}{I_{\mathrm{LCP}}^{\text {blue }}+I_{\mathrm{RCP}}^{\text {blue }}+I_{\mathrm{LCP}}^{\text {red }}+I_{\mathrm{RCP}}^{\text {red }}} .
\end{aligned}
$$

We refer to $M^{\vartheta}$ as the magnetogram signal. $M^{\vartheta}$, as defined above, can be written as

$M^{\vartheta}=\alpha\left(B, T, v_{\mathrm{LOS}}\right) B^{\vartheta}$.

The magnetogram signal is proportional to the longitudinal magnetic field $B^{\vartheta}$, but it is also a function of $B$ itself, the temperature $T$, and the line-of-sight (LOS) velocity $v_{\mathrm{LOS}}$. The magnetogram bias $(M \equiv 0)$ is calculated as the mean magnetogram signal from a reference region showing undisturbed granulation outside the sunspot. For our analysis we use only magnetograms obtained in the blue wing of Fe I, because red wing data is only available for a few data sets. In Figs. 1 to 4, magnetograms and their corresponding continuum references are shown. To calibrate the Dopplergrams (i.e., to estimate the calibration function $f_{\mathrm{c}}$ ) we scanned the iron line with the SOUP filter using a step size of $1 \mathrm{pm}$ in a quiet region outside the sunspot (cf. Fig. 5, left panel). By reading off intensities at two wavelengths separated by $10 \mathrm{pm}$, corresponding to the separation of the wavelengths for the red- and blue-wing magnetograms, as a function of the average of the two wavelengths, and combining the measurements as in Eq. (2), the calibration function $f_{\mathrm{c}}$ could be estimated (cf. Fig. 5, solid curve in the right panel). The sign convention in $f_{\mathrm{c}}$ is selected such that positive velocities represent red shifts. This calibration only allows a rough estimate of Doppler velocities since it relies on the assumption that the line profile is the same in the sunspot penumbra as in the adjacent quiet sun. Based on this calibration we calculate rms velocity fluctuations of about $900 \mathrm{~m} \mathrm{~s}^{-1}$ in the region of reference showing undisturbed granulation outside the sunspot. This is about $200 \mathrm{~m} \mathrm{~s}^{-1}$ higher than values obtained by other observers who used spectral information from comparable heights (e.g. Hirzberger et al. 2001). This difference may be due to the higher spatial resolution of our data.

\subsection{Magnetic and flow field geometry}

For symmetrical spots, LOS magnetograms and Dopplergrams can be used to analyze vector magnetic fields and flows by performing measurements over many penumbral filaments and assuming that their properties vary only with the distance from the center of the sunspot. In the following, this method is sketched for the magnetic field, the same analysis is valid for the flow field (e.g. Schlichenmaier \& Schmidt 2000).

To investigate the geometry of the magnetic field (cf. Fig. 6) as a function of the radial distance from sunspot centre $r$ and the azimuth angle $\varphi$ the following assumptions are made: (i) the magnetic field vector $\boldsymbol{B}$ has no azimuthal component within the parts of the penumbrae analyzed. As a consequence the measured azimuthal variation in $\boldsymbol{B}$ is purely a line-of-sight effect; 

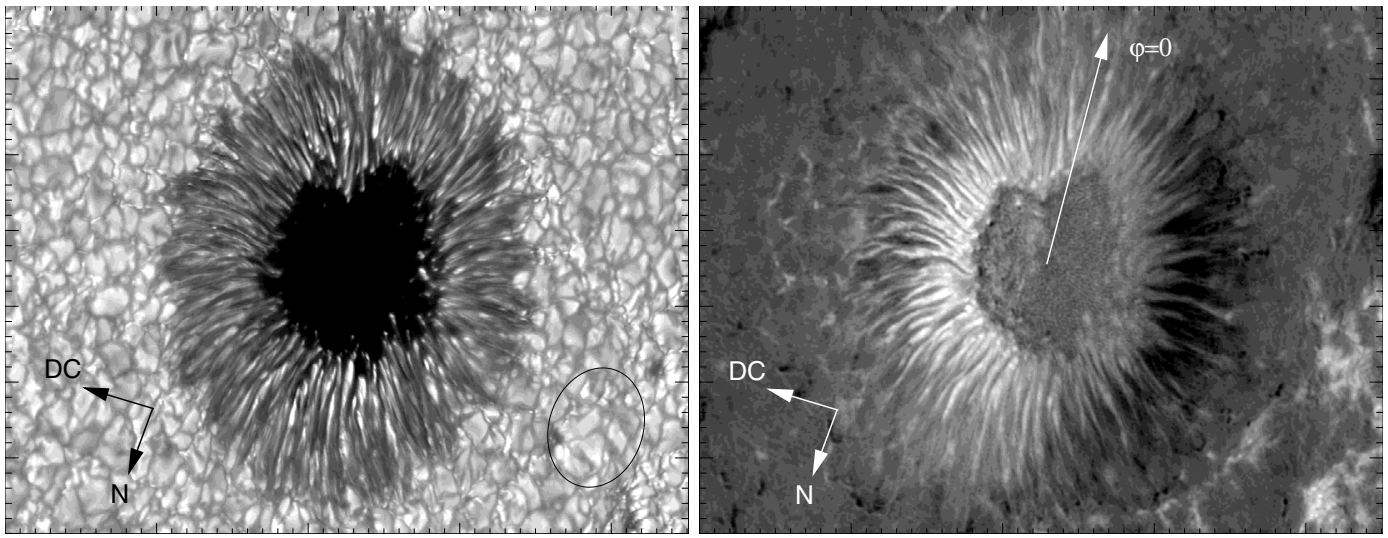

Fig. 1. Data from August 4, 2003, NOAA 10425, $\mu=0.76$. Left: reference image, right: magnetogram. DC - Disk center, N - solar north. The ellipse plotted in the reference image shows the projection of a circle at a LOS angle of $39^{\circ}$. Minor tickmarks are $1^{\prime \prime}$ apart.
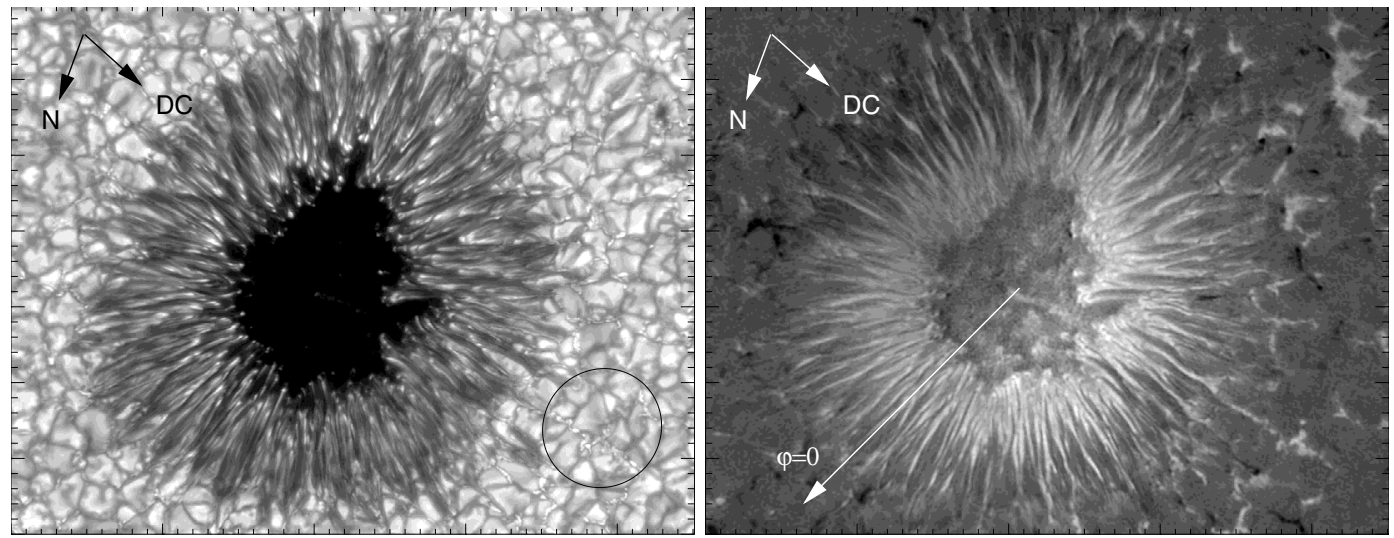

Fig. 2. Data from August 8, 2003, NOAA 10425, $\mu=0.96$. Left: reference image, right: magnetogram. DC - Disk center, N - solar north. The ellipse plotted in the reference image shows the projection of a circle at a LOS angle of $16^{\circ}$. Minor tickmarks are $1^{\prime \prime}$ apart.

(ii) the general structure of the magnetic field does not change with time, which makes it possible to average observations that are obtained on different days.

The azimuth angle $\varphi$ is defined such that $\varphi=90^{\circ}$ points to disk center. The angle of inclination $\gamma$ is measured relative to the normal of the solar surface. With assumption (i) above, the LOS component of the magnetic field is described by

$B^{\vartheta}(r, \varphi)=B_{0}(r)[\sin \varphi \sin \vartheta \sin \gamma(r)+\cos \vartheta \cos \gamma(r)]$

where $B_{0}$ is the magnetic field strength of the field line inclined by $\gamma$ to the normal of the surface. Using this equation, we rewrite Eq. (3) for the magnetogram signal as

$$
\begin{aligned}
M^{\vartheta}(r, \varphi)= & \alpha\left(B(r), T(r, \varphi), v_{\mathrm{LOS}}(r, \varphi)\right) \\
& \times B_{0}(r)[\sin \varphi \sin \vartheta \sin \gamma(r)+\cos \vartheta \cos \gamma(r)]
\end{aligned}
$$

The projection of the horizontal component of $\alpha \boldsymbol{B}$ to the LOS

$$
\begin{aligned}
M_{\mathrm{h}}^{\vartheta}(r)= & \alpha\left(B(r), T(r, \varphi), v_{\mathrm{LOS}}(r, \varphi)\right) \\
& \times B_{0}(r) \sin \varphi \sin \vartheta \sin \gamma(r) \\
= & M_{\mathrm{h}, 0}^{\vartheta}(r) \sin \varphi
\end{aligned}
$$

describes an azimuthal sinusoidal variation for constant $r$ with the amplitude

$M_{\mathrm{h}, 0}^{\vartheta}(r) \equiv \alpha\left(B(r), T(r, \varphi), v_{\mathrm{LOS}}(r, \varphi)\right) B_{\mathrm{h}, 0}^{\vartheta}(r)$.
The projection of the vertical component of $\alpha \boldsymbol{B}$ to the LOS $M_{\mathrm{v}}^{\vartheta}(r)$ does not vary with the azimuth and is represented by the offset of the sinusoidal least square fit to $M^{\vartheta}(r, \varphi)$ for constant $r$. The inclination angle $\gamma$ is computed from the ratio of $M_{\mathrm{v}}^{\vartheta}(r)$ and $M_{\mathrm{h}, 0}^{\vartheta}$. This ratio gives a good estimate of the inclination of the magnetic field vector because the factor $\alpha$ cancels out:

$\arctan \left(\frac{M_{\mathrm{h}, 0}^{\vartheta}(r)}{M_{\mathrm{v}}^{\vartheta}(r) \tan \vartheta}\right)=\arctan \left(\frac{B_{\mathrm{h}, 0}^{\vartheta}(r)}{B_{\mathrm{v}}^{\vartheta}(r) \tan \vartheta}\right) \equiv \gamma$.

The accuracy of the derived magnitudes $M_{\mathrm{h}}^{\vartheta}, M_{\mathrm{v}}^{\vartheta}, M_{0} \equiv \alpha B_{0}$ and $\gamma$ depends mainly on the filtergram quality obtained in the line wing of the Fe I-line at $630.2 \mathrm{~nm}$. The fact that the filtergrams in left and right polarized light are not obtained simultaneously represents the main error source for our analysis. Photon and read-out noise are negligible in comparison to the artifacts that result from the subtraction of filtergram data obtained during varying seeing conditions. Therefore we restrict the analyzed magnetogram data to the very best filtergrams and do not attempt a quantitative error analysis. Another critical part of the analysis is the zero point calibration of the magnetograms and the Doppler maps. Errors in this calibration have a major effect on the accuracy in the estimate of $M_{\mathrm{v}}^{\vartheta}$ and consequently also $\gamma$ and $M_{0}$. The estimate of $M_{\mathrm{h}}^{\vartheta}$ is independent of this offset but sensitive to the quality of the least squares fit for the azimuthal sinusoidal variation. 

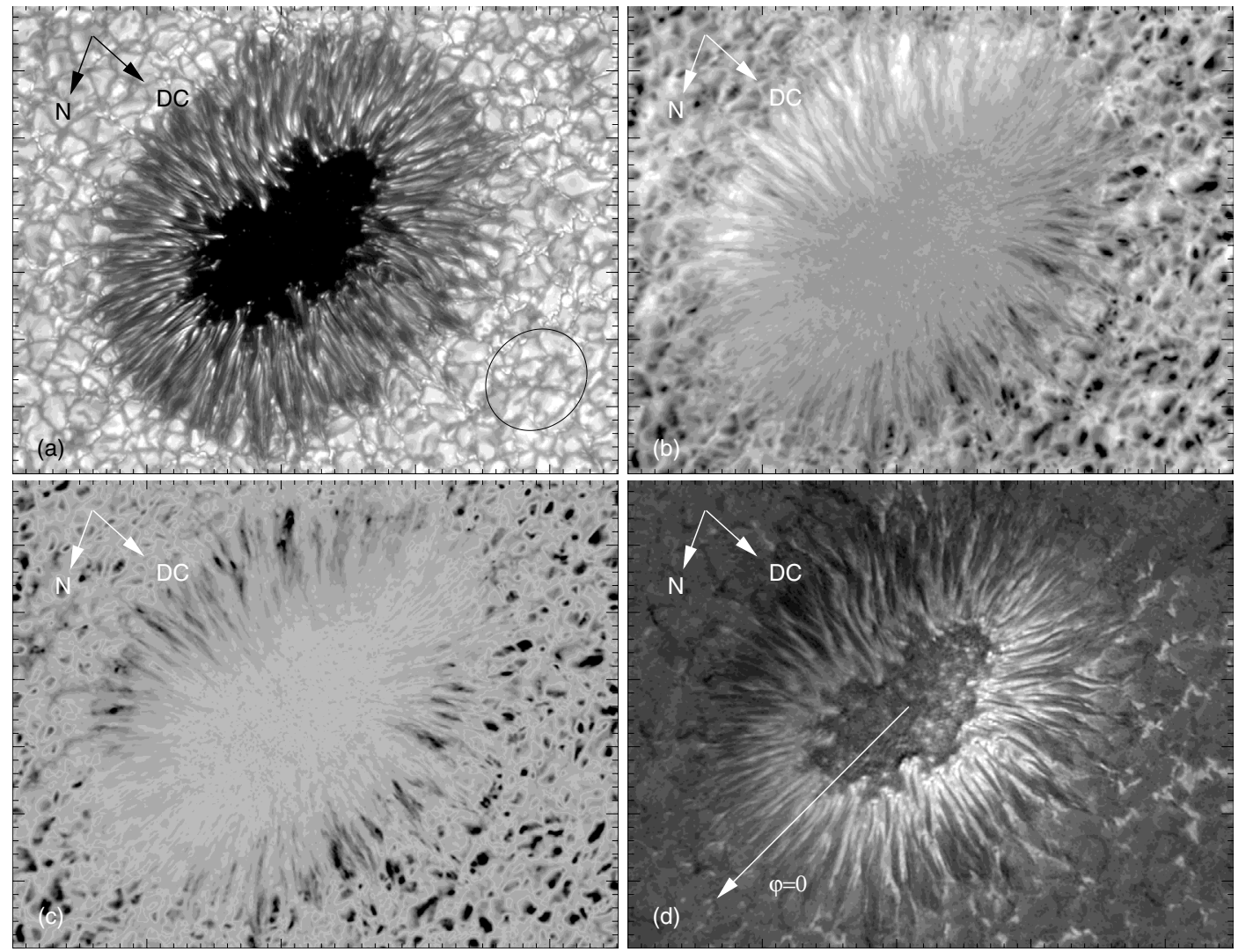

Fig. 3. Data from August 9, 2003, NOAA 10425, $\mu=0.88$. a) Reference image; b) Dopplergram, range $-3000 \mathrm{~m} \mathrm{~s}^{-1}$ to $3000 \mathrm{~m} \mathrm{~s}^{-1}$; c) corrector map, range 0.6 to 1.3 ; d) corrected magnetogram. DC - Disk center, $\mathrm{N}$ - solar north. The ellipse plotted in the reference image shows the projection of a circle at a LOS angle of $28^{\circ}$. Minor tickmarks are $1^{\prime \prime}$ apart.

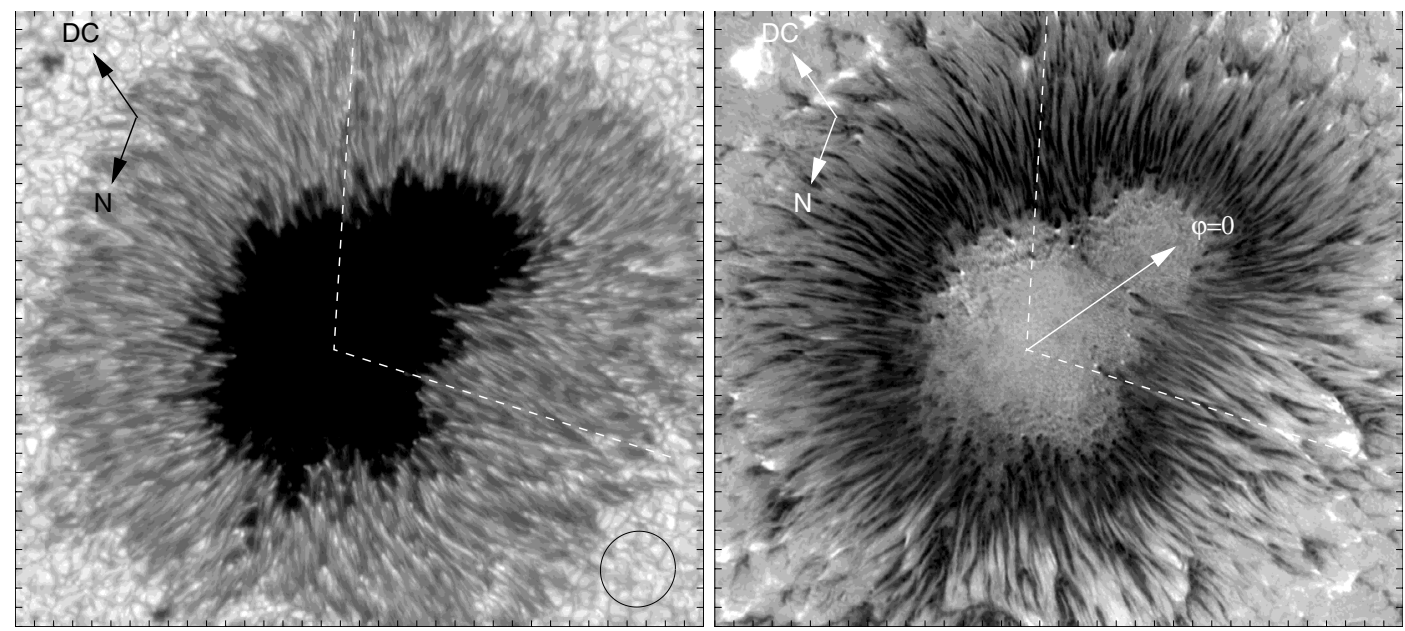

Fig. 4. Data from Jul. 2, 2003, NOAA 10397, $\mu=0.97$. Left: reference image, right: magnetogram. DC - Disk center, $\mathrm{N}-$ solar north. The ellipse plotted in the reference image show the projection of a circle at a LOS angle of $12^{\circ}$. Minor tickmarks are $2^{\prime \prime}$ apart. This spot has opposite polarity to that of NOAA 10425 . For clarity the magnetogram signal is inverted for the analysis.

\subsection{Influence of velocity fields on the magnetogram signal}

To investigate the effect of LOS velocities on the measurement of the longitudinal magnetic field, we used polarimetric data of the penumbra of NOAA 10425 (August 9), obtained with the Polarimetric Littrow Spectrograph at the German Vacuum Tower Telescope on Tenerife, Spain (Beck et al. 2005). Both the Stokes $I$ and the Stokes $V / I$ profile for the penumbra are plotted in the left panel of Fig. 7. To obtain an estimate for the magnetogram signal we shifted the obtained Stokes $V / I$ profile corresponding to Doppler shifts in a range between -3.5 and $3.5 \mathrm{~km} \mathrm{~s}^{-1}$. The normalized values (cf. Eq. (1)) at line center $\pm 5 \mathrm{pm}$ are plotted in the right panel of Fig. 7. The black and gray curves refer to measurements in the blue and red wings of the Fe I $630.2 \mathrm{~nm}$ line, respectively. The diagram illustrates the 

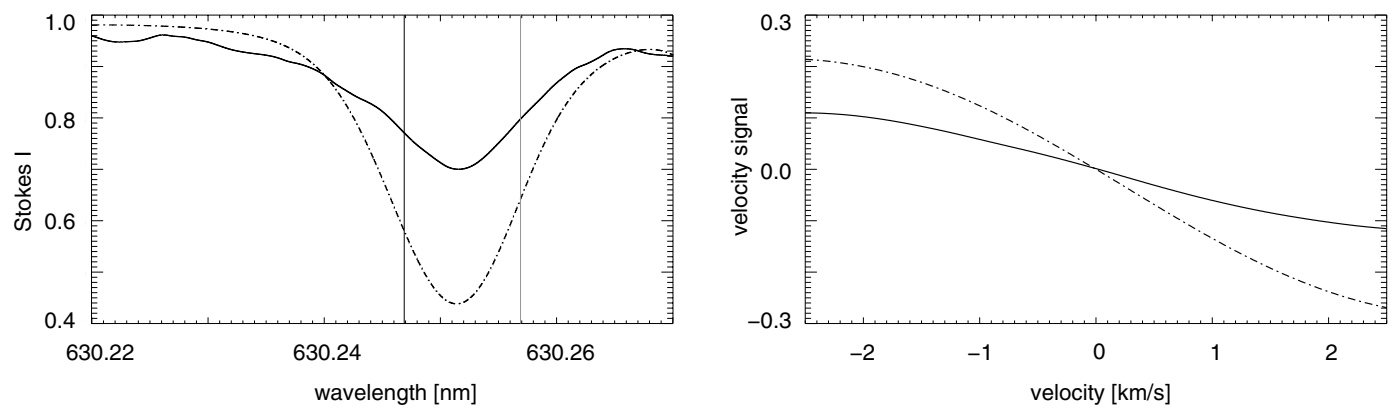

Fig. 5. Left: line scan of Fe I $630.2 \mathrm{~nm}$. The solid curve is the measured line profile (interpolated) and the dashed dotted curve is the atlas profile convolved with a simple transmission profile with $F W H M$ value corresponding to that of the SOUP filter. The two vertical lines correspond to the nominal (for zero Doppler shift) wavelengths of the recorded magnetograms in the blue and red wings (line center position \pm 5 pm). Right: velocity calibration for the measured line profile (solid) - used to define the calibration function $f_{\mathrm{c}}$ - and the convolved atlas profile (dashed-dotted).

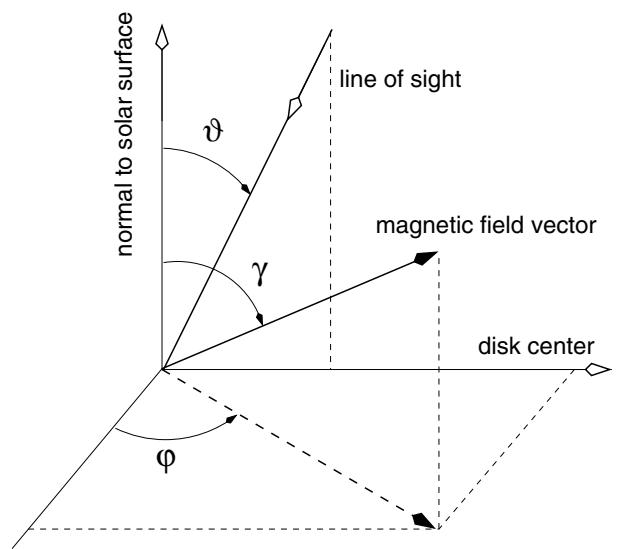

Fig. 6. Field geometry. The azimuth angle $\varphi$ is defined such that $\varphi=90^{\circ}$ points to disk center. The angle of inclination $\gamma$ and the heliocentric angle $\vartheta$ are measured relative to the normal of the solar surface.

sensitivity of the magnetogram signal for Doppler velocities. The measurement error of the magnetogram signal increases rapidly with increasing absolute value of the LOS velocity. Already relatively small LOS velocities limit the measurement of the magnetogram signal. Using the information of this diagram, magnetograms of data sets that include Dopplergrams can be corrected for the influence of a present velocity field. An example for a corrected magnetogram is shown in Fig. 3 together with the corresponding Dopplergram, the reference image, and the corrector map. The LOS velocities have a relatively small effect on the derivation of the magnetic inclination angle. For our analysis the magnetograms are not corrected, but a comparison of the results based on a corrected and an uncorrected magnetogram respectively is discussed in Sect. 4.3.

\subsection{Temperature dependence of the magnetogram signal}

To estimate the temperature dependence of the magnetogram signal, radiative transfer calculations were made using the temperature and electron pressure stratifications of the mean penumbral model of del Toro Iniesta et al. (1994). The calculations are based on the following assumptions: (i) the magnetic field strength $(1200 \mathrm{G})$, the field inclination $\left(60^{\circ}\right)$ and the azimuth $\left(0^{\circ}\right)$ are height independent; (ii) the macro-turbulence is assumed to be $0 \mathrm{~km} \mathrm{~s}^{-1}$, because of the high spatial resolution of the observations; (iii) no LOS velocities are considered (cf. Sect. 3.3).

The radiative transfer calculations were made in 160 different atmospheres. They differ only in the temperature stratification $T_{n}$, which is constructed as

$T_{n}(\tau)=T_{\mathrm{TTR}}(\tau)+\Delta T, \quad-2000 \mathrm{~K}<\Delta T<+1000 \mathrm{~K}$

at all heights, where $T_{\mathrm{TTR}}$ is the temperature stratification of the model of del Toro Iniesta et al. (1994). The temperature at $\tau=1$ in this model is only $200 \mathrm{~K}$ lower than the quiet sun temperature at $\tau=1$. Before computing the Stokes $I$ and $V$ profiles for each atmosphere, the electron pressure stratification was recalculated to maintain hydrostatic equilibrium. This was done by solving the ionization equilibrium for the temperatures of the model. Finally, the calculated Stokes $I$ and $V$ profiles were convolved with a simple transmission profile (Lorentz shape) with a $F W H M$ of 7.2 pm centered at Fe I $630.2 \mathrm{~nm}-5 \mathrm{pm}$ from line center. From these profiles a simulated magnetogram signal as obtained through the SOUP filter was calculated.

Figure 8 shows the variation of this magnetogram signal with the temperature $\Delta T$. The magnetogram signal changes by less than $\pm 20 \%$ in the range of $\Delta T$ from $-1700 \mathrm{~K}$ to $+400 \mathrm{~K}$. We note that the magnetogram signal increases if the atmosphere cools down a bit with respect to the mean penumbral temperature stratification $T_{\mathrm{TTR}}$.

\subsection{Coordinate system}

The penumbral boundaries were defined by continuum intensity levels indicating the transition from the umbra to the penumbra $\left(I=0.38 I_{0}\right)$ and penumbra to the granulation $\left(I=0.85 I_{0}\right)$ - denoted by white and black contour lines, respectively, in Fig. 9. $I_{0}$ is defined as the averaged continuum intensity in the reference region showing undisturbed granulation outside the sunspot. All data points between these contour lines belong to the penumbra and form an individual penumbral mask for each data set. Rather than trying to trace out azimuthal paths parallel to the penumbral boundaries, the contours of the 

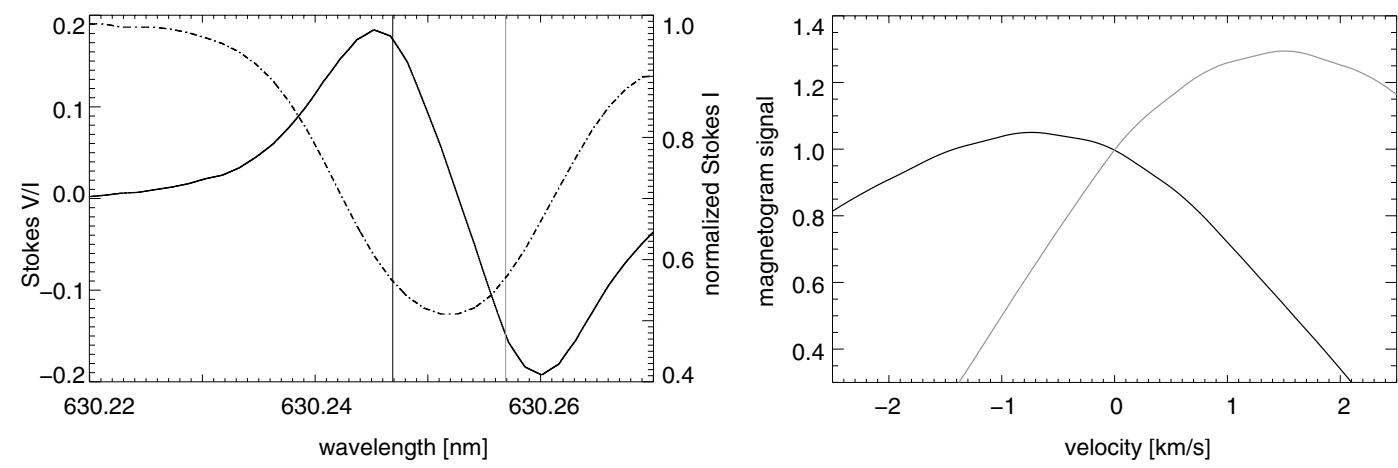

Fig. 7. Influence of a velocity field on the magnetogram signal. Line indication as in Fig. 5. Left: stokes $V / I$ (solid) and Stokes $I$ (dashed-dotted, scale to the right) averaged over the penumbra of NOAA 10425 (August 9), obtained with the Polarimetric Littrow Spectrograph at the German Vacuum Tower Telescope on Tenerife. Right: variation in magnetogram signal vs. LOS velocity. Black and gray curves refer to measurements in the blue and red wings of the Fe I $630.2 \mathrm{~nm}$ line, respectively.

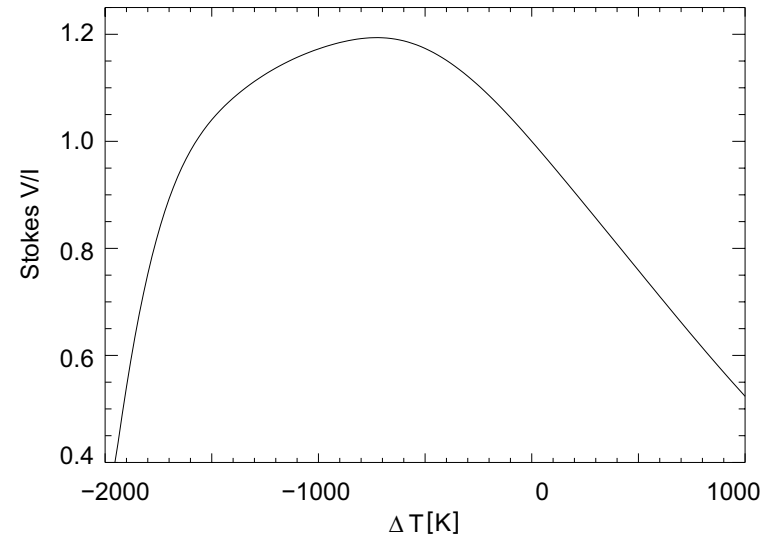

Fig. 8. Temperature dependence of the magnetogram signal. The Stokes $V / I$ signal is normalized to the value of Stokes $V / I$ that corresponds to the mean penumbral temperature stratification of del Toro Iniesta et al. (1994) $(\Delta T=0)$.

inner and outer penumbral boundaries were fitted by ellipses. The parameters of these ellipses were interpolated such that each data point in the penumbra belongs to a uniquely defined ellipse corresponding a projected circle with the radius $r$ given by the semi-major axis. In this manner the inner and outer radii of the penumbra were defined as the semi-major axes of the ellipses for which at least $80 \%$ of the data points are located within the penumbral boundaries, defined above. To facilitate inter-comparisons between different spots, the radial coordinate $r$ was normalized to the outer radius of the penumbra. The azimuth angle $\varphi$ is defined such that $\varphi=90^{\circ}$ is pointing to disk center.

On July 2, the spot in AR 10397 developed a bulge in the upper right quadrant (cf. Fig. 4) and a segment (indicated by the dashed lines) was excluded from the analysis.

\subsection{Filament masks}

To distinguish different components of penumbral fine structure, two types of masks are defined. Both masks separate lowand high-signal components of the penumbral fine structure, measured relative to a locally defined average signal. The first
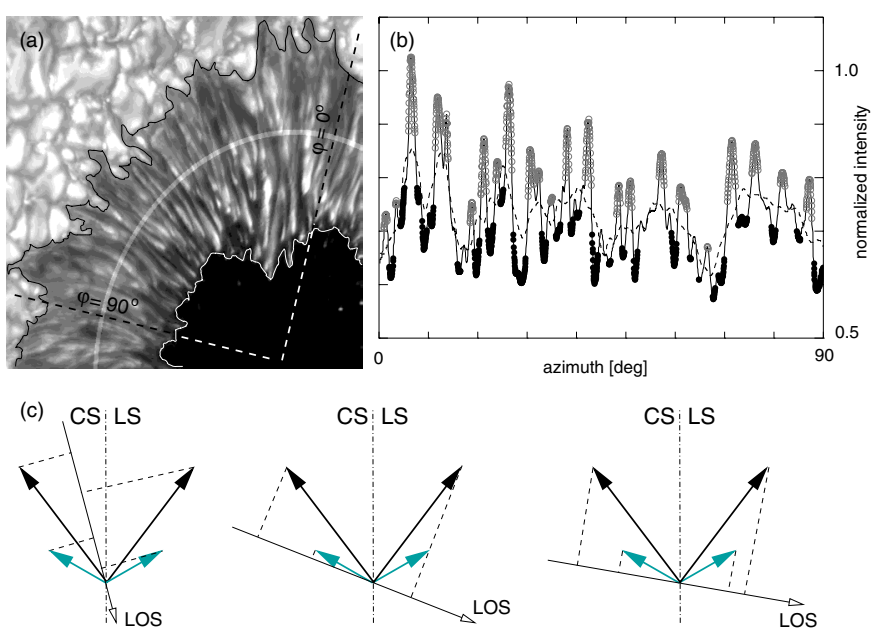

CS: $B_{\text {LOS }}>B_{\text {LOS }}$ LS: $B_{\text {LOS }}>B_{\text {LOS }}$

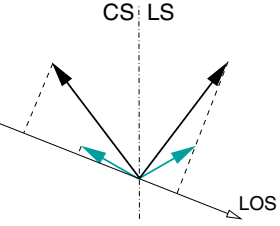

CS: $B_{\text {LOS }}>B_{\text {LOS }}$

LS: $B_{\text {LOS }} \approx B_{\text {LOS }}$

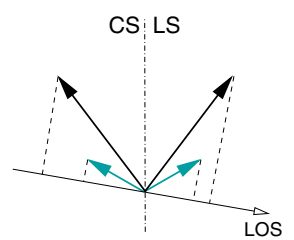

CS: $B_{\text {LOS }}>B_{\text {LOS }}$
Fig. 9. Separation of dark and bright filaments based on the broadband continuum data. a) Spot segment where a ring of constant distance from spot center is high-lightened. The black solid line indicates the boundary of the penumbra toward the granulation, the white line the boundary toward the umbra. b) The solid curve illustrates the azimuthal intensity variation along the ring, including all data points. The dashed curve denotes the locally defined cut-off-intensity. The data points that belong to the bright components are marked by open circles, the data points that belong to the dark components by solid circles. c) Illustration of the validity of magnetogram-signal-related masks. CS - center-side, LS - limb-side. See text for discussion.

type of mask is based on local values of continuum intensity and selects structures which are traditionally described as dark and bright filaments. The second type of mask is based on the magnetic signal and selects local low-signal and high-signal features in the magnetograms. The calculations of the masks that are based on the continuum intensity are illustrated in Fig. 9. The penumbra is divided in rings $r+\Delta r$ of constant radius $r$ to spot center $\left(\Delta r=0{ }^{\prime} 16\right)^{1}$. The cut-off intensity (dashed curve in Fig. 9) is calculated by the intensity average at each point over a ring segment defined by the width $\Delta r$ and

1 There is an inconsistency in that $\Delta r$ is constant on the image plane and not deprojected to the solar horizontal plane. 
$\Delta \varphi=4.8^{\circ}$. In order to obtain well-defined masks, the transitions between the two components are excluded. This also excludes intensity variations on very small spatial scales - scales that are not resolved in the magnetograms or the $630.2 \mathrm{~nm}$ continuum images.

Applying this method to the magnetograms is more critical because the signal varies with both temperature $T$, LOS velocity $v_{\mathrm{LOS}}$, inclination of the field vector, and the field strength. In the magnetogram of Fig. 3, obtained at a heliocentric angle of $28^{\circ}$, the azimuthal variation of the magnetogram signal is distinct. In addition to this variation we observe a local variation on the spatial scale of penumbral filaments. A locally strong magnetogram signal alternates with a weak magnetogram signal. A definition of a magnetic-field related mask is therefore possible. As will be discussed in detail later, observations at other heliocentric angles show that the inclination in the part of the penumbra selected by the low-magnetogramsignal mask is greater than that selected by the high-signal mask. Given this, we can expect the definition of a magnetogram mask to become problematic at some LOS angle when the magnetogram signal from both penumbral components will be similar. This is the case for the inner limb-side penumbra, observed at a LOS angle of $39^{\circ}$, the largest heliocentric position observed (cf. Fig. 1). If the LOS angle were even larger, the magnetogram signal of the limb-side penumbra would become inverted in comparison to the signal of the center-side penumbra. Figure 9c illustrates this problem for the same magnetic field configuration at three different heliocentric angles. The left panel illustrates the case where the mask based on the magnetogram signal is well defined. In the center panel the measured magnetogram signal of the two components become similar in the limb-side penumbra. The right panel presents the case wherein the definition of magnetogram masks fails; such a mask would not select the same kind of penumbral structure on the limb- and the center-side of the penumbra.

\section{Results}

\subsection{General observations}

In Fig. 10 parts of the center-side penumbrae of both the small symmetric and the larger less symmetric sunspot are displayed at the same image scale. The extension of the penumbra of the small spot is about $10^{\prime \prime}$. The filamentary structures stretch over the whole penumbra. Details visible in the continuum image, in particular the dark cores of penumbral filaments (Scharmer et al. 2002; Sütterlin et al. 2004), can easily be identified in the magnetogram. The penumbra of the larger spot extends over $20^{\prime \prime}$ and is more irregular in the outer part. Penumbral filaments seem to originate not only inside the boundary between the umbra and the penumbra but also at larger distances from spot center. As a consequence, the azimuthal average at a specified distance from spot center contains contributions from different parts of the individual filaments. Especially in the outer penumbra, filamentary structures originate locally and fan out with increasing distance from spot center. In addition, the outer penumbra appears more fuzzy than the inner region in the continuum image. Here it is more difficult to identify small

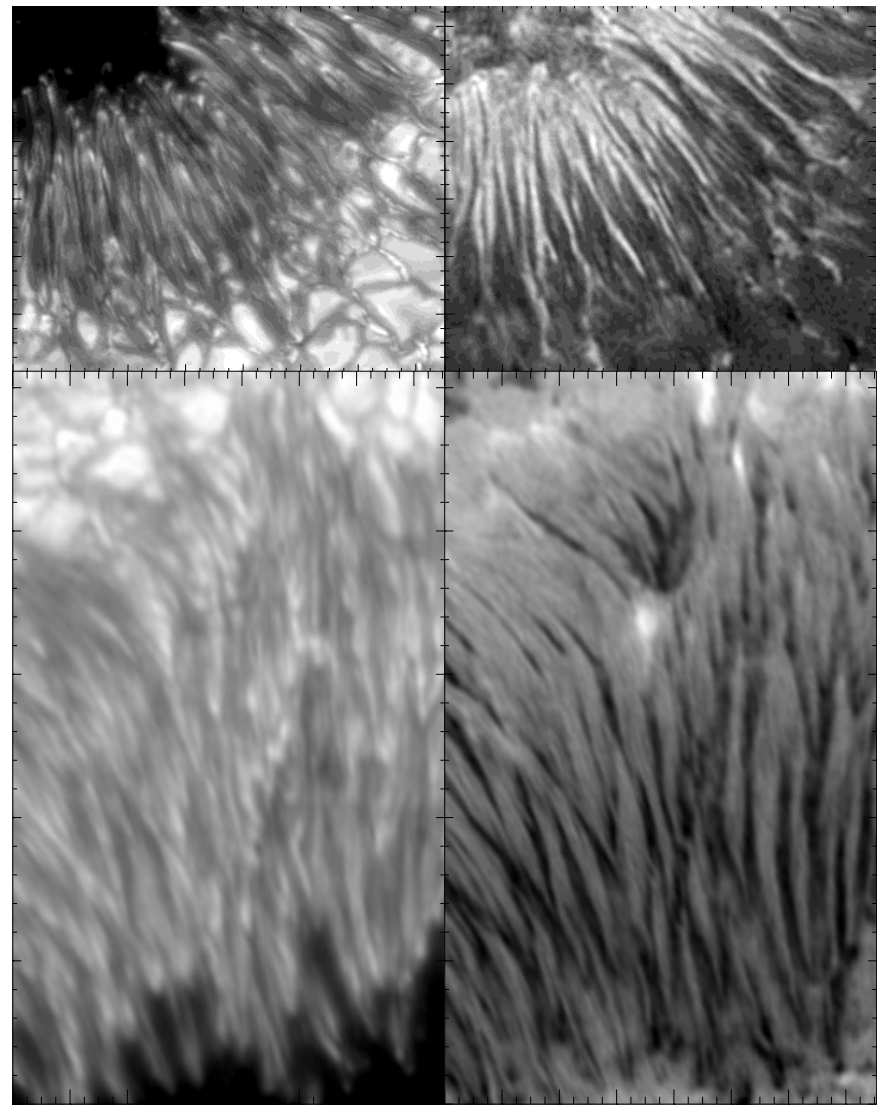

Fig. 10. Top: center-side penumbra (REF, MB) of the symmetric sunspot (NOAA 10425, LOS angle $16^{\circ}$, August 4), Bottom: centerside penumbra (REF, MB) of the large less symmetric sunspot (NOAA 10397, LOS angle $12^{\circ}$, July 2 ). (The large less symmetric sunspot has opposite polarity to that of NOAA 10425.) Distance between minor tickmarks corresponds to 0.5 .

structures and to distinguish between "dark" and "bright" filamentary structures. This leads to a less reliable identification of the different components of the penumbra from the segmentation masks than is the case for the inner and middle penumbra.

The filamentary structures are very stable on a time scale of at least $1.5 \mathrm{~h}$. Figure 11 shows eight snap shots of a small part of the center-side penumbra of the symmetric sunspot of NOAA 10425 on August 8 at $\mu=0.97$. This sequence demonstrates the long life time of this magnetic structure. The selected dark core - marked by the dashed line in the first continuum image - stretches over the whole penumbra during the whole observed sequence. During $1.5 \mathrm{~h}$ this dark-cored filament showed very little evolution. It moved about one arcsecond sideways and toward the umbra.

\subsection{Azimuthal slices}

To analyze the azimuthal variation of the magnetogram and Dopplergram data the penumbra is again divided in rings $r+\Delta r$ with $\Delta r=0$ ' 16 . Figure 12 shows the LOS magnetogram signal of two individual data pairs as a function of the azimuth, measured at three different radii, at the inner $(r=0.45)$, the 


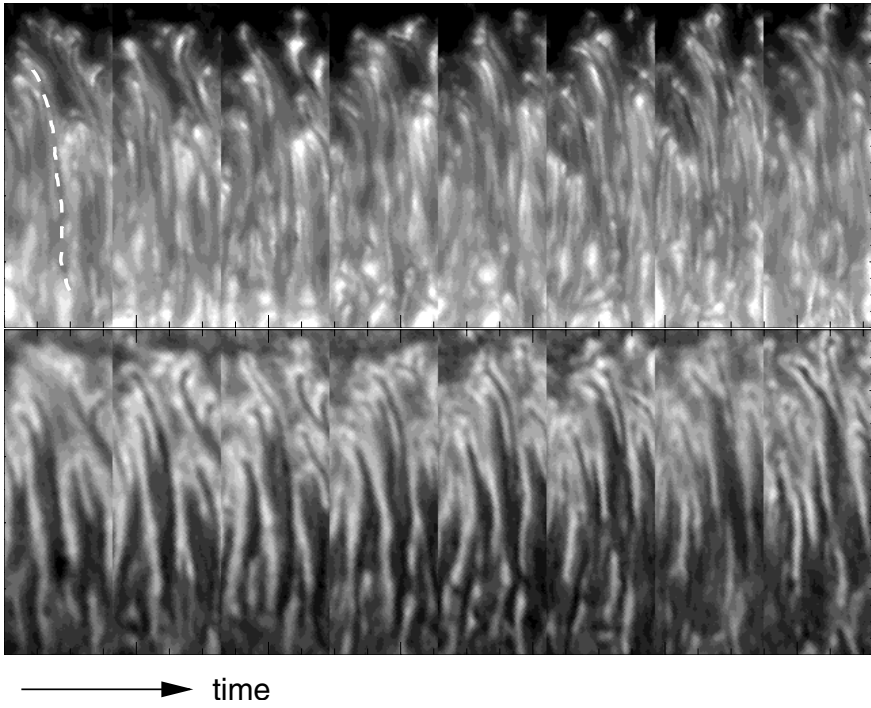

Fig. 11. Evolution of dark-cored filaments during $1 \mathrm{~h} 35 \mathrm{~min}$ in the $\mathrm{G}$ continuum (top) and magnetogram (bottom). One tickmark corresponds to $1^{\prime \prime}$.

$\operatorname{mid}(r=0.70)$ and the outer penumbra $(r=0.95)$. The overall sinusoidal shapes clearly demonstrate the presence of a horizontal magnetic field component. To determine the amplitude of the azimuthal variation, $M_{\mathrm{h}, 0}$, a sinusoidal least squares fit was performed to the data points weighted by their measurement errors. The free parameters for this fit are the bias and the amplitude of the sine function. The vertical component of the LOS signal is represented by the offset obtained from the sinusoidal least square fit (horizontal lines in Fig. 12). In the plots of Fig. 12 the gray dots refer to the bright component, as selected by the continuum intensity mask. Black dots refer to the dark component. There is not an exact correlation between the brightness and the magnetogram signal even if in most cases the dark components of filaments are related to local minima of the magnetogram signal. The small arrows in the third panel of Fig. 12 indicate examples of local correlation (gray) and anticorrelation (black).

For both the bright and dark component, as selected by the continuum intensity mask, the azimuthal variation of the magnetogram signal is stronger for larger heliocentric angle and most obvious in the plots representing the mid penumbra. The vertical LOS component, $M_{\mathrm{v}}^{\vartheta}$, decreases continuously with increasing distance from spot center. For the radii $r=0.45$ and $r=0.70$ at both heliocentric angles $M_{\mathrm{v}}^{\vartheta}$ of the bright structures is larger than the amplitude of the sinusoidal. For the dark component $M_{\mathrm{v}}^{\vartheta}$ reaches zero in the outer penumbra. That indicates a horizontally oriented magnetic field within structures that appear locally dark in the continuum image. In the inner penumbra the amplitude of the azimuthal variation is lower than in the mid penumbra, while the vertical LOS component, $M_{\mathrm{v}}^{\vartheta}$, decreases. This indicates smaller inclination angles in the inner part of the penumbra for both the bright and dark components.

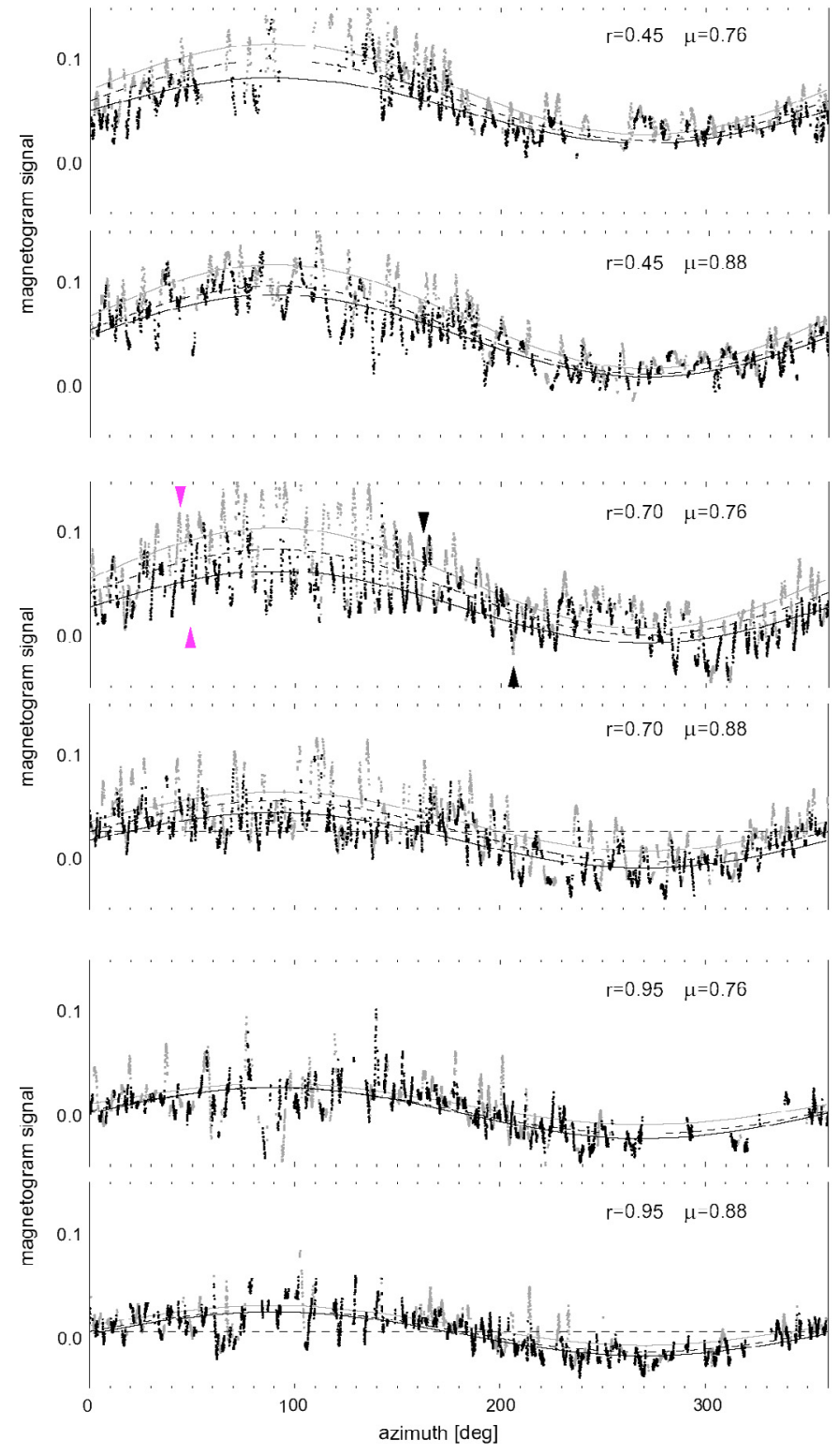

Fig. 12. Azimuthal slices for NOAA 10425 on August 4, 2003 $(\mu=0.76)$ and August 9, $2003(\mu=0.88)$ : inner penumbra $r=0.45$, mid penumbra $r=0.70$ and outer penumbra $r=0.95$. Gray and black dots refer to the bright and dark component, respectively, as selected by the continuum intensity mask. The sinusoidals, denoted by gray curves, show the fits which take only the data points of the dark components into account. The black sinusoidals refer to the bright components and the dashed sinusoidal is based on all data points. The horizontal lines, representing the vertical LOS component, are indicated in the same way.

\subsection{Magnetic field inclination}

Using Eq. (8) we calculate the inclination angle $\gamma$ of the magnetic field. To obtain the radial dependence of the strength of the magnetogram signal we also calculate $M_{\mathrm{h}} \equiv M_{\mathrm{h}}^{\vartheta} / \sin \vartheta$, $M_{\mathrm{v}} \equiv M_{\mathrm{v}}^{\vartheta} / \cos \vartheta$ and $M_{0}$. The same analysis was made for the existing Doppler maps (cf. Table 1). Figure 13 shows the radial dependence of the normalised continuum intensity (a) and the inclination angle of the magnetic field (d). The diagrams Figs. 13b, c show the qualitative variation of the horizontal and 

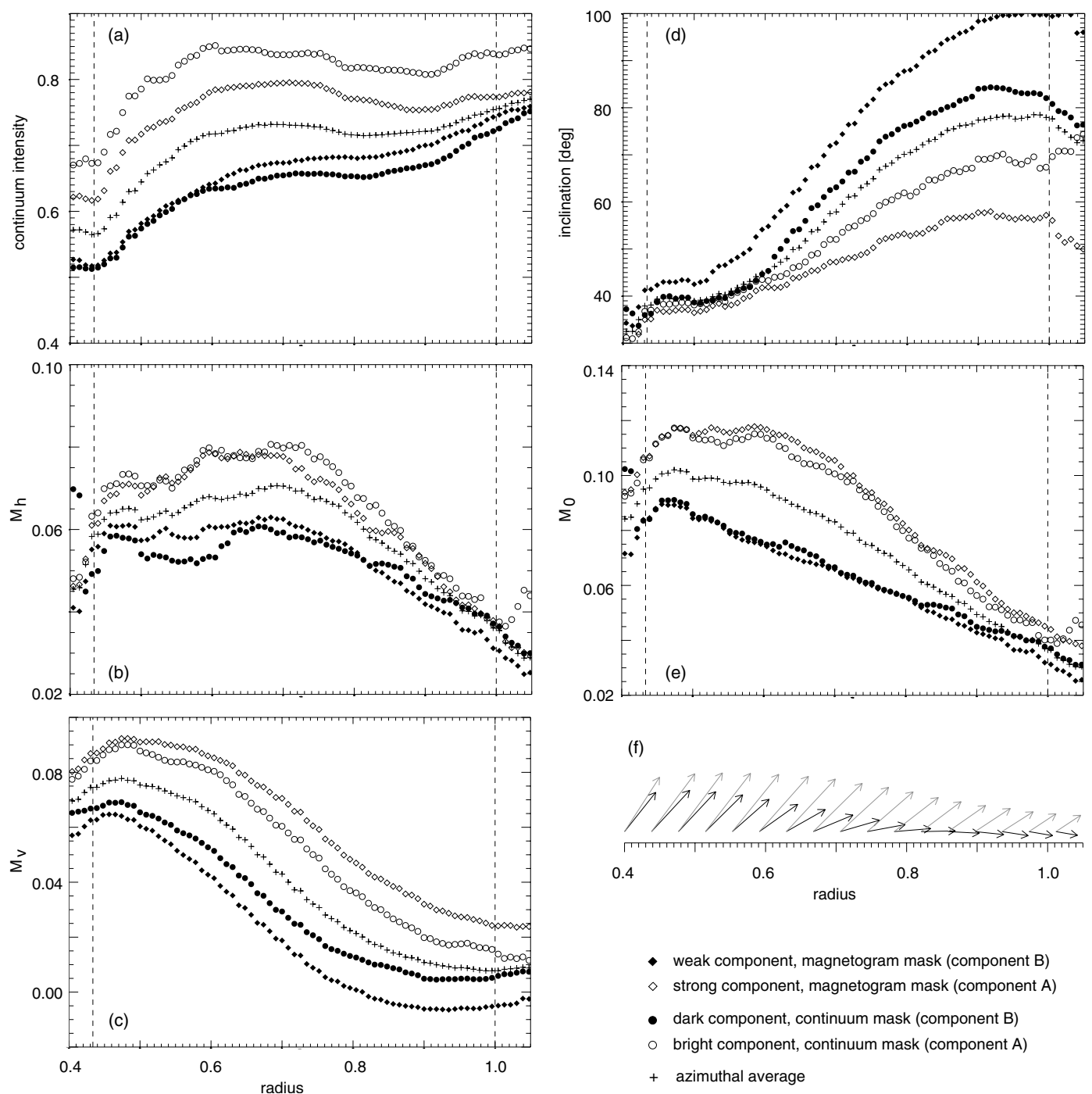

Fig. 13. Average radial variation of the magnetic field magnitudes based on all data sets of the symmetric sunspot, NOAA 10425, observed on August 4, 8 and 9. a) Continuum intensity, normalized to quiet sun intensity; b) horizontal component of the magnetogram signal (arbitrary units); c) vertical component of the magnetogram signal; d) inclination of the magnetic field; e) absolute strength of the magnetogram signal; f) vector plot of magnetic field from the data of $\mathbf{d}$ ) and e). Black arrows represent the penumbral component B as selected by the magnetogrambased mask, gray arrows component A. Vertical dashed lines show the limits of umbra and penumbra. The symbols in the diagrams a)-e) refer to data points selected by the different types of mask as described in Sect. 3.6 and indicated in the lower right. The pluses correspond to selecting all data points at a given radius.

vertical component of the magnetogram signal. In Fig. 13e the variation of the absolute strength of the magnetogram signal is plotted. The diagrams represent the average radial variation based on all data sets of the symmetric sunspot, observed on August 4, 8 and 9. The curves in Fig. 13 cannot be used to infer $B_{h}, B_{v}$ and $B_{0}$ due to the unknown temperature stratification. However, these curves can be used as constraints on theoretical penumbral models by calculation of synthetic SOUP magnetograms, as described previously, and for comparison with our observed data.

The result of this analysis is the following: the average inclination of the magnetic field, as obtained by selecting all data points at a given radius, increases from approximately $40^{\circ}$ in the inner penumbra to about $70^{\circ}$ in the outer penumbra. This radial dependence of the azimuthally averaged inclination angle is in agreement with earlier results (e.g. Title et al. 1993; Keppens \& Martinez Pillet 1996).

By repeating the analysis for data points selected by the two magnetic masks, we can identify two spatially separated components of the penumbral magnetic field orientation: the weak component - denoted by solid diamonds in Fig. 13 - is characterized by a rapidly increasing inclination angle toward the outer penumbra, reaching horizontal orientation at $r \sim 0.80$ and pointing downward in the outer penumbra. The inclination angle for the strong component - denoted by open diamonds - reaches only $60^{\circ}$ in the outer penumbra. In the inner part of the penumbra $(0.45<r<0.55)$, the inclination angle is approximately $35-45^{\circ}$ for both components.

When the analysis is repeated for the data points selected by the continuum-based mask, the two resulting components 


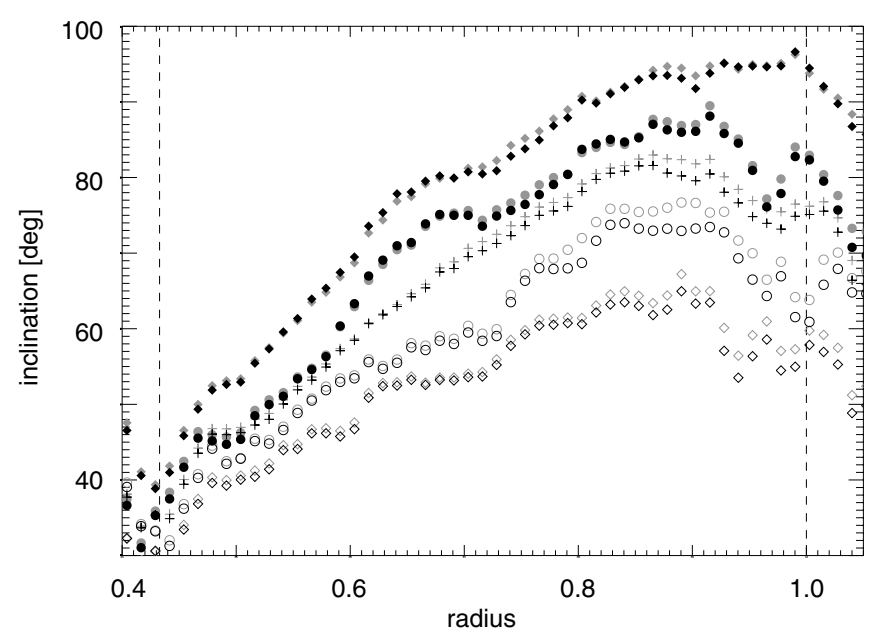

Fig. 14. Single measurement of magnetic field inclination of the small circular sunspot (Aug. 4, UT 14:25). Dark symbols refer to the calculation of $\gamma$ based on a magnetogram that is corrected for the influence of the flow field, the gray symbols in the background to the calculation based on the uncorrected magnetogram (cf. Sect. 3.3).

show the same behaviour, albeit somewhat less pronounced. We therefore conclude that on the average, the dark parts of the penumbral filaments are related to the more horizontally orientated magnetic field. Thus it seems reasonable to introduce provisional designations for these two penumbral components - A and $\mathrm{B}$ - where the magnetogram signal for the $\mathrm{B}$ component (Fig. 13e) is significantly weaker than for the A component. Analyzing the horizontal component, $M_{\mathrm{h}}$, and the vertical component of the magnetogram signal, $M_{\mathrm{v}}$, separately, we find that $M_{\mathrm{h}}$ varies by a relatively small amount in the inner and middle penumbra for both the A and the B components, with a possible slight increase for the A component in the mid penumbra. Beyond approximately $r=0.70$, the strength of $M_{\mathrm{h}}$ decreases linearly with radius to approximately $50 \%$ of the maximum value in the outer penumbra. In contrast, the strength of $M_{\mathrm{v}}$ (Fig. 13d) decreases continuously with radius over the whole penumbra. This results in an almost linear decrease of the absolute strength of the magnetogram signal (Fig. 13e) starting at $r=0.45$ for the B component and at $r=0.60$ for the A component. For the A component the continuum intensity shows a maximum in the mid penumbra $(0.55<r<0.75)$. This brightening is not visible for the B component but coincides with an increase of $M_{\mathrm{h}}$. Figure $13 \mathrm{f}$ summarizes the results in a vector plot. Black arrows refer to the B component as selected by the magnetogram-based mask, gray arrows to the A component.

The diagram in Fig. 14 displays the radial dependence of inclination of a single measurement for NOAA 10425 (Aug. 4, UT 14:25). The result is based on a magnetogram that is corrected for the influence of the flow field (cf. Sect. 3.3). The gray symbols in the background denote the result based on the uncorrected magnetogram. As can be seen, Doppler shifts have only a marginal effect on the derived inclination angles.

Figure 15 shows the radial variation of the magnetic inclination of the large less symmetric sunspot (NOAA 10397), observed on July 1, 2 and 5. The variation of the average inclination, based on all data points, with radius (Fig. 15) is the

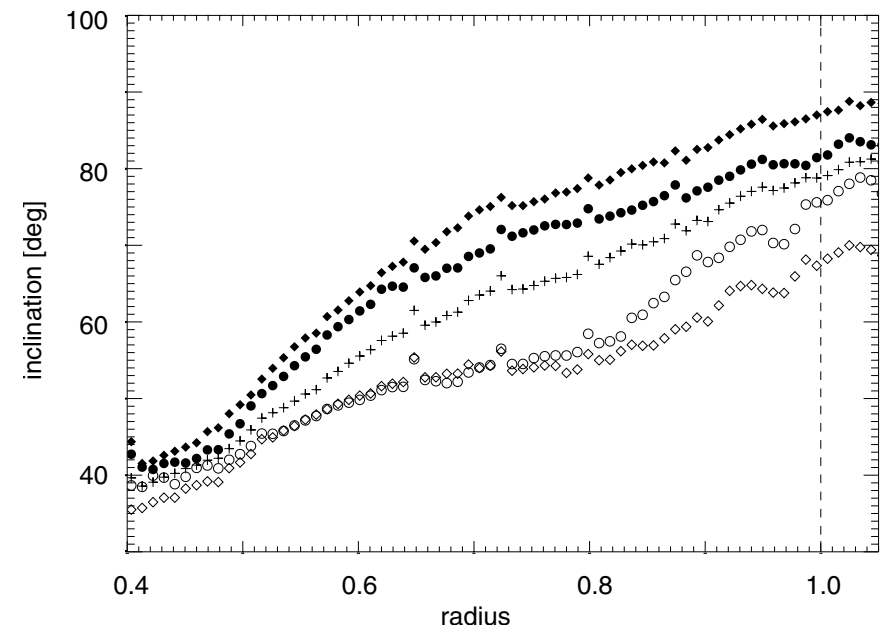

Fig. 15. Results for NOAA 10397. Inclination of the magnetic field, based on data observed on July 1,2 and 5. Continuum intensity related data only based on July 2 and 5 .

same as for the small symmetric sunspot (NOAA 10425). The inclination angle $\gamma$ increases from $40^{\circ}$ in the inner penumbra to $70^{\circ}$ at the outer edge of the penumbra. For the larger sunspot, the splitting into two components with different inclination takes place at a lower value of the normalized radius $(r \sim 0.50)$. However, measuring the absolute distance in arcseconds from the inner penumbral boundary, the separation into a more horizontal and a more vertical component takes place at the same distance of about two arcseconds from the inner penumbral boundary. The difference in inclination between the two components is not as large as for the smaller sunspot, the peak difference being only approximately $25^{\circ}$ whereas for the smaller sunspot it is $45^{\circ}$. This difference might be explained by the more irregular structure of the penumbra of the large spot (cf. Sect. 4.1).

The correlation diagrams in Fig. 16 show that the two magnetic field components behave differently toward the outer penumbral border. The absolute strength of the magnetogram signal of the A component decreases almost linearly with increasing inclination. For both the $\mathrm{A}$ and the $\mathrm{B}$ components, the absolute strength of the magnetogram signal peaks for inclination angles close to $40^{\circ}$. But in contrast to the case of the A component, the absolute strength of the magnetogram signal of the B component decreases rapidly for small inclination angles $\left(40^{\circ}<\gamma<45^{\circ}\right)$ and more slowly for larger angles. In addition, the plots show a distinct "hook" for data points with low absolute strength of the magnetogram signal (cf. Westendorp Plaza et al. 2001). Very close to the umbra, the signal weakens even further even though the magnetic field most probably gets stronger, so this may well be a saturation effect.

\subsection{Velocity field inclination}

Measurements of the flow field inclination are difficult with this data because of uncertainties in the absolute velocity calibration. Rather than attempting to use the surrounding photosphere as reference and applying a correction for the 


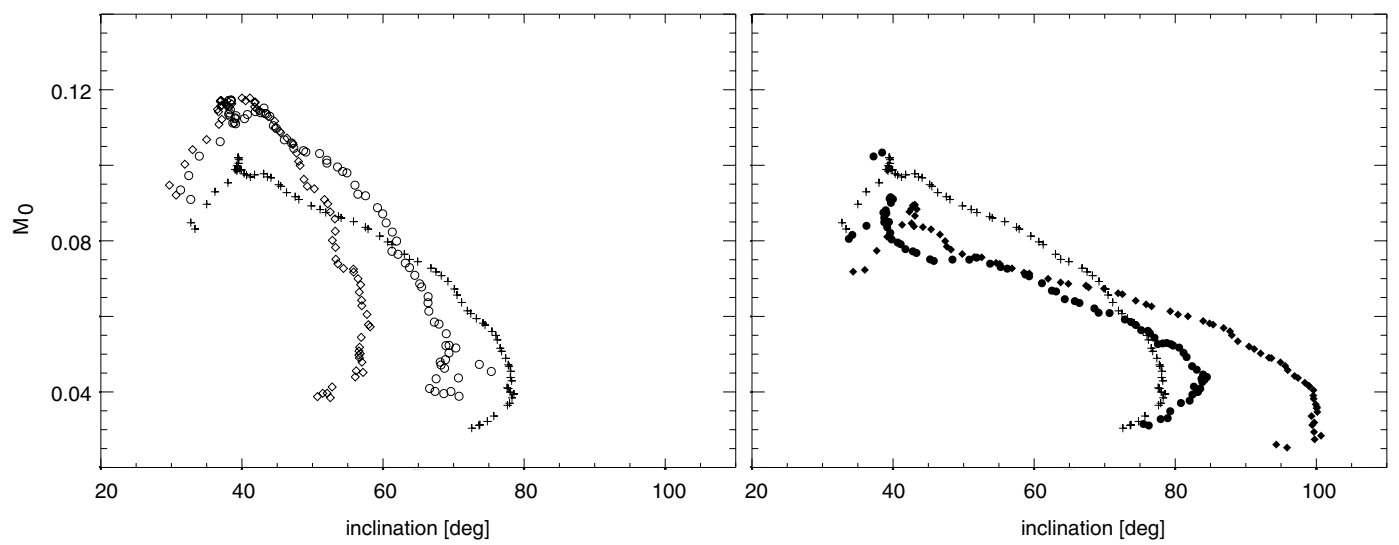

Fig. 16. Correlation diagrams: magnetic field vs. inclination angle. (Diagrams based on NOAA 10425 data.) Left: high-signal components, right: low-signal components. Symbols as in Fig. 13.
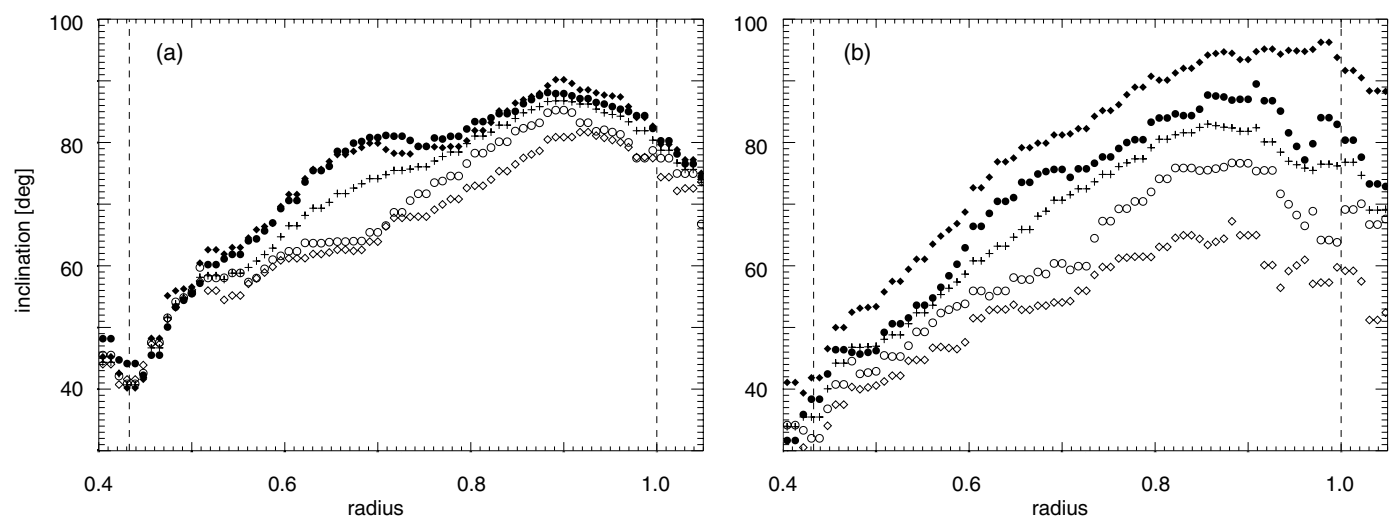

Fig. 17. Comparison of the flow field and magnetic field inclination of the symmetric sunspot, NOAA 10425, August 9, 2003. a) Inclination angle of the flow field; b) inclination angle of the magnetic field. Vertical dashed lines show the limits of umbra and penumbra. Symbols are explained in Fig. 13.

convective blue shift, we have chosen to build the calibration on the assumption of the umbra being at rest in spite of the low signal-to-noise ratio of the filtergram signal in the umbra and the uncertainty due to scattered light from the penumbra into the umbra. If so, this calibration introduces some additional uncertainties due to molecular blends that occur in the umbra around the Fe I-line at $630.2 \mathrm{~nm}$ (cf. Lites et al. 1998). This is in any case the only useful method for the large spot, because it covers nearly the entire FOV. Further, two-wavelength Dopplergrams are very sensitive to the wavelength offsets from line center. Only filtergram data that are obtained at essentially the same offset from line center in the red and blue wings can be used for Doppler measurements, otherwise the variation of the source function with height introduces crosstalk which gives spurious Doppler signals.

We restrict the analysis of the flow-field geometry to the discussion of two single measurements, keeping in mind the uncertainties mentioned above. Figure 17a shows the variation of the velocity field inclination, and Fig. 17b shows the magnetic field inclination of the symmetric sunspot in NOAA 10425. Given that the estimate of the inclination for the velocity is less accurate than for the magnetic field, we draw the following tentative conclusions: (a) the inclination of the velocity field increases from the inner to the outer part of the penumbra; (b) the velocity field of the penumbral B component is more horizontal than that of the A component; (c) the radial variations of the inclinations of the velocity field and the magnetic field show detailed similarities. For example, the inclination angle of both the velocity field and the magnetic field remains constant between $r=0.60$ and $r=0.70$ for the A component of the symmetric sunspot. The absolute flow velocity increases linearly from the inner penumbra toward the outer penumbra and peaks at $r=0.90$ for both field components (not plotted).

The maximum inclination of the azimuthally averaged flow field is $85^{\circ}$, reached at $r=0.90$. This is close to a horizontal orientation and is in disagreement with the results of Schlichenmaier \& Schmidt (2000), who found downflows for the azimuthal averaged flow field in the outer penumbra.

\section{Summary and discussion}

We first note that an analysis that ignores fine structure in the azimuthal direction and includes all measured values of the polarization signal gives a variation in the inclination of the magnetic field that increases from $40^{\circ}$ in the inner to almost $80^{\circ}$ in the outer penumbra. This variation is in good agreement with former observations (e.g. Title et al. 1993; Keppens \& Martinez Pillet 1996). 
The data presented here are unique for their high spatial resolution of approximately 0 '.15-0.'20. At this resolution, the magnetic field appears structured in the form of two essentially radial components with large differences in inclination angle. As discussed in Sect. 3.2 the errors are dominated by seeing-induced artefacts that are very difficult to analyze quantitatively. However, we refer to the consistency of the results we obtain from data sets obtained on different days as strong evidence that our results are valid.

We describe the penumbra as consisting of two components, one of which is characterized by having a more vertical magnetic field - the A component - and one having a more horizontal field - the B component. The A component forms coherent structures that extend across the entire penumbra, some of them forming branches, giving the impression of vertical sheet-like structures.

The observed main characteristics of the A component are: (a) The magnetic inclination angle increases from $40^{\circ}$ in the inner to $60^{\circ}$ in the outer penumbra. (b) It is related to the bright parts of penumbral filaments as selected by locally high values of the continuum intensity. (c) The strength of the magnetogram signal is stronger than that of the $\mathrm{B}$ component throughout the penumbra. The difference in strength of the magnetogram signal appears to peak in the middle penumbra and decrease toward the outer penumbral boundary. (d) The related flow velocities are lower than for the B component. The difference in flow velocities is less than $700 \mathrm{~m} \mathrm{~s}^{-1}$. (e) The continuum intensity is enhanced in the mid penumbra $(0.55<$ $r<0.75)$. This enhancement coincides with an increase of the strength of the horizontal component of the magnetogram signal.

The B component is characterized by the following: (a) the inclination angle increases rapidly with increasing distance from spot center; (b) the inclination angle ranges from $40^{\circ}$ to $100^{\circ}$. The magnetic field is essentially horizontal outside $r=0.80$; (c) this component is related to locally dark filamentary structures in continuum intensity; (d) the B component is associated with a flow field with azimuthally averaged velocities of up to $2.5 \mathrm{~km} \mathrm{~s}^{-1}$. The peak flow velocity is reached at the radius where both the magnetic and the velocity field are approximately horizontal.

The innermost part of the penumbra is irregular with a few individual filaments protruding well into the umbra. Because of this, our method of inferring the inclination and strength of the magnetogram signal from azimuthal variations cannot be used. In addition, the signal of the filaments in this region must be contaminated by the umbral structures. However these filaments also show variations in magnetogram signal across their widths that are consistent with those measured further out in the penumbra, suggesting significant variations in field strength and/or magnetic field inclination. The overall measured variations in inclination angle for the magnetic field are small in the inner penumbra. The separation into the $\mathrm{A}$ and $\mathrm{B}$ components takes place at $r=0.60$ for the small symmetric spot, and at $r=0.50$ for the large less symmetric spot. These radii correspond to the same absolute distance of about $2^{\prime \prime}$ from the inner penumbral edge. In this $2^{\prime \prime}$ wide region, the inclination of both components is very similar and show only small variations in the radial direction.

The magnetic inclination is clearly related to the continuum intensity of the filaments, even though correlation is not exact. One explanation for this is most likely that the 436.39-nm continuum intensity and the $630.25 \mathrm{~nm}$ Stokes $V$ signal are formed at different heights and therefore cannot be expected to correlate perfectly. The observed anti-correlation at some locations may also be caused by the different spatial resolution obtained in the 436.39-nm continuum and the SOUP filtergram images, which are furthermore not recorded strictly simultaneously.

The A component shows a local maximum in the continuum intensity in the mid penumbra that coincides with an increase of the horizontal component of the magnetogram signal. This bright ring - restricted to the bright filaments - can hardly be seen in the azimuthal average over the whole penumbra. To date, the most convincing proof of the existence of a bright ring in the mid penumbra has been presented by Westendorp Plaza et al. (2001) from the inversion of magnetically sensitive lines.

In previous investigations, the flow field is generally found to be more horizontal than the average vector magnetic field by roughly $10^{\circ}$ (e.g. Adam \& Petford 1991; Solanki 2003, for a review), implying that material motions do not occur along the lines of magnetic force. Bellot Rubio et al. (2003, 2004) appear to solve this problem in their interpretation of full Stokes profiles by allowing two magnetic atmospheres to coexist in one resolution element. They find that one of the components is associated with a flow that is well aligned with the magnetic field. This result, obtained from inversion of spectro-polarimetric data at low spatial resolution, agree well with our results based on high-resolution magnetograms. Borrero et al. (2004) show that similar results can also be obtained from inversions based on a one-component atmosphere, where all physical parameters are allowed to vary with height, and emphasize that the models are significantly different from the geometrical point of view.

Our observations suggest rapid variations in inclination of the magnetic field and flow-field over small distances and that the flow-field of the more inclined B component is roughly aligned with the magnetic field. Our analysis therefore confirms the existence of a "fluted" or "uncombed" magnetic field in the penumbra. In addition, all highly resolved magnetograms obtained near disk center show dark-cored filaments as prominent features. The magnetogram signal is lower in the dark (cooler) cores than in the lateral (warmer) brightenings. This is opposite to the effect shown in Fig. 8 which predicts a higher magnetogram signal at lower temperatures $(-1500 \mathrm{~K}<\Delta T<$ $0 \mathrm{~K}$ ) with respect to the mean penumbral temperature stratification. Our data therefore suggests that the magnetic field strength must be weaker in the dark cores, or possibly that the inclination of the magnetic field is larger than for the lateral brightenings. Further investigation of this requires full vector spectro-polarimetry at spatial scales at 0.2 or below.

The usage of longitudinal magnetograms limits our investigation to the interpretation of the magnetogram signal. The variation of the magnetic field strength remains uncertain and needs to be investigated with the help of spectropolarimetric data. Speculating that the observed magnetogram signal is proportional to the magnetic field strength we draw 
the following conclusions. As regards the variation of the average strength of the magnetogram signal with radius, the analysis made here is in reasonable agreement with former measurements (Keppens \& Martinez Pillet 1996; Westendorp Plaza et al. 2001) except in the inner penumbra where saturation effects may occur. The continuum intensity and the magnetic field strength show a positive correlation in the sense that the magnetic field strength is weaker for the dark penumbral structures. This is in agreement with some (e.g. Wiehr \& Stellmacher 1989), but not all (e.g. Beckers \& Schröter 1969; Abdussamatov 1976), earlier investigations, based on data with much lower spatial resolution. Our results suggest that the azimuthal fluctuations of the magnetic field are not caused by inclination fluctuations alone, as suggested by Title et al. (1993), but by combined fluctuations of inclination and field strength as found by later investigators (cf. Martinez Pillet 1997).

The present observations are in broad agreement with the two-component models of Bellot Rubio et al. (2003, 2004) based on spectro-polarimetric data made at low spatial resolution. Within the framework of these models, the flux tubes could be identified as the dark components (including the dark penumbral cores) of the penumbral filaments, surrounded by a brighter component having a more vertical magnetic field. There is an overall agreement between these models and our data regarding the identification of one component of the penumbra having weaker, more horizontal magnetic field and a strong flow roughly aligned with the magnetic field - our $\mathrm{B}$ component. It is also interesting to note that dark penumbral cores are clearly visible in the inner penumbra and visible also in the middle penumbra but difficult to identify in the outer penumbra except for very small sunspots.

Bellot Rubio et al. (2004) remark that their analysis suggests that the flux tubes are located at increasingly deeper layers in the outer penumbra. Quantitatively, there are significant differences between our results and those of Bellot Rubio et al. (2003, 2004). Their analysis suggests that the magnetic field of the component carrying the bulk of the Evershed flow is nearly horizontal already at $r=0.5$ and has a very small variation in field strength with radius. Our analysis indicates that the magnetic field is much more vertical in the inner penumbra and shows a significant variation in magnetogram signal throughout the penumbra. Clearly the small scale of, e.g., dark cores (about $90 \mathrm{~km}$ ) implies that spatial stray-light could strongly degrade our measurements.

Our data may also be consistent with models that contain a flux tube with horizontal magnetic field embedded in a more vertical magnetic field, as discussed by Solanki \& Montavon (1993), Martínez Pillet (2000) and Borrero et al. (2004). However, such models need to further develop realistic models of the magnetic field surrounding the flux tube in order to allow comparisons with observations. The existence of long and long-lived, nearly horizontal, flux tubes (cf. Fig. 11) also poses the question of how the energy needed to balance the radiative output is transported to the surface (cf. Schlichenmaier \& Solanki 2003).

The MISMA inversion (cf. Sánchez Almeida 2005b,a) suggests that high resolution magnetograms in sunspots close to solar disk center should show both positive and negative polarities. We do not find significant evidence for this in our magnetograms. Possibly, the resolution of the magnetograms is not sufficiently high to detect these opposite polarities. The recently developed image restoration technique by van Noort et al. (2005), MFBD with multiple objects and phase diversity, yields magnetograms with better resolution and less artefacts than the ones presented here. Future use of this technique for sunspot analyses promises to improve upon the results presented here.

The B-components of the penumbra are associated with a more inclined magnetic field that is also related to weaker magnetogram signal and lower intensity than that of the surroundings. Spruit (2005) has pointed out that such magnetic fields may be the surface manifestation of field-free gaps, associated with magneto-convection, just below the visible surface. Spruit (2005) argues that such gaps will be associated with a magnetic field that to a first approximation will be a potential field surrounding the field-free gap and shows that this magnetic field will tend to be weaker and more horizontal above the gap, as observed. His model avoids the need for penumbral magnetic fields that carry any currents (except in a thin sheet), and thus also torques, as opposed to the fluted model of Title et al. (1993).

The discovery of dark penumbral cores of Scharmer et al. (2002) suggests that we are starting to resolve fundamental scales in sunspot penumbrae. Spectro-polarimetric data that are sensitive to the magnetic field both close to the photosphere and well above the photosphere, and obtained at a spatial resolution approaching 0 '! 1 may therefore allow us to draw firm conclusions about the fine scale organization of penumbral magnetic fields.

Acknowledgements. The Swedish 1-m Solar Telescope is operated on the island of La Palma by the Royal Swedish Academy of Sciences in the Spanish Observatorio del Roque de los Muchachos of the Instituto de Astrofísica de Canarias. K. Langhans' research is funded by the European Community's Human Potential Program through the European Solar Magnetism Network (contract HPRN-CT-2002-00313). We are grateful to the referee, L. Bellot Rubio. His remarks greatly improved the paper and he generously provided the radiative transfer data needed to investigate the temperature dependence of the magnetogram signal. C. Beck generously offered his polarimetric data of NOAA 10425 to estimate the influence of present velocity fields on the magnetogram signal. B. Lites and J. Sánchez Almeida are thanked for valuable comments on the manuscript. Students of the Nordic Baltic Research School on Observational astrophysics assisted with the observations on July 5: J.-E. Ovaldsen, E. Lindfors, A. Järvinen and S. Joki-Huuki. J. Darelid, summer student at the SST, assisted with the observations in August.

\section{References}

Abdussamatov, K. I. 1976, Sol. Phys., 48, 117

Adam, M. G., \& Petford, A. D. 1991, Sol. Phys., 135, 319

Beck, C., Schmidt, W., Kentischer, T., \& Elmore, D. 2005, A\&A, accepted

Beckers, J. M. 1968, Sol. Phys., 3, 258

Beckers, J. M., \& Schröter, E. H. 1969, Sol. Phys., 10, 384

Bellot Rubio, L. R., Balthasar, H., \& Collados, M. 2004, A\&A, 427, 319 
Bellot Rubio, L. R., Balthasar, H., Collados, M., \& Schlichenmaier, R. 2003, A\&A, 403, L47

Berger, T. E., \& Title, A. M. 2001, ApJ, 553, 449

Berger, T. E., Rouppe van der Voort, L. H. M., Löfdahl, M. G., et al. 2004, A\&A, 428, 613

Borrero, J. M., Solanki, S. K., Bellot Rubio, L. R., Lagg, A., \& Mathew, S. K. 2004, A\&A, 422, 1093

Danielson, R. E. 1961a, ApJ, 134, 275

Danielson, R. E. 1961b, ApJ, 134, 289

Degenhardt, D. 1989, A\&A, 222, 297

Degenhardt, D. 1991, A\&A, 248, 637

Degenhardt, D., \& Wiehr, E. 1991, A\&A, 252, 821

del Toro Iniesta, J. C., Tarbell, T. D., \& Ruiz Cobo, B. 1994, ApJ, 436, 400

Hirzberger, J., \& Kneer, F. 2001, A\&A, 378, 1078

Hirzberger, J., Koschinsky, M., Kneer, F., \& Ritter, C. 2001, A\&A, 367,1011

Jahn, K. 1992, in NATO ASIC Proc., Sunspots: Theory and Observations, 375, 139

Johannesson, A. 1993, A\&A, 273, 633

Keppens, R., \& Martinez Pillet, V. 1996, A\&A, 316, 229

Kinman, T. D. 1952, MNRAS, 112, 425

Lites, B. W., Skumanich, A., \& Scharmer, G. B. 1990, ApJ, 355, 329

Lites, B. W., Elmore, D. F., Seagraves, P., \& Skumanich, A. P. 1993, ApJ, 418, 928

Lites, B. W., Thomas, J. H., Bogdan, T. J., \& Cally, P. S. 1998, ApJ, 497, 464

Lites, B. W., Socas-Navarro, H., Skumanich, A., \& Shimizu, T. 2002, ApJ, 575, 1131

Löfdahl, M. G. 2003, in Image Reconstruction from Incomplete Data II, ed. P. J. Bones, M. A. Fiddy, \& R. P. Millane, Proc. of the SPIE, 4792, 146

Martinez Pillet, V. 1997, in 1st Advances in Solar Physics Euroconference, Advances in Physics of Sunspots, ASP Conf. Ser., 118,212

Martínez Pillet, V. 2000, A\&A, 361, 734

Mathew, S. K., Lagg, A., Solanki, S. K., et al. 2003, A\&A, 410, 695

Rimmele, T. R. 1995, A\&A, 298, 260

Rouppe van der Voort, L. H. M. 2002, A\&A, 389, 1020

Rouppe van der Voort, L. H. M., Löfdahl, M. G., Kiselman, D., \& Scharmer, G. B. 2004, A\&A, 414, 717

Sánchez Almeida 2005a, ApJ, submitted

Sánchez Almeida 2005b, ApJ, 622, 1292
Sütterlin, P., Bellot Rubio, L. R., \& Schlichenmaier, R. 2004, A\&A, 424, 1049

Scharmer, G. B., Bjelksjo, K., Korhonen, T. K., Lindberg, B., \& Petterson, B. 2003a, in Innovative Telescopes and Instrumentation for Solar Astrophysics, ed. Stephen L. Keil, \& Sergey V. Avakyan, Proc. of the SPIE, 4853, 341

Scharmer, G. B., Dettori, P. M., Löfdahl, M. G., \& Shand, M. 2003b, in Innovative Telescopes and Instrumentation for Solar Astrophysics, ed. Stephen L. Keil, \& Sergey V. Avakyan, Proc. of the SPIE, 4853, 370

Scharmer, G. B., Gudiksen, B. V., Kiselman, D., Löfdahl, M. G., \& Rouppe van der Voort, L. H. M. 2002, Nature, 420, 151

Schlichenmaier, R., Jahn, K., \& Schmidt, H. U. 1998a, ApJ, 493, L121

Schlichenmaier, R., Jahn, K., \& Schmidt, H. U. 1998b, A\&A, 337, 897

Schlichenmaier, R., \& Schmidt, W. 2000, A\&A, 358, 1122

Schlichenmaier, R., \& Solanki, S. K. 2003, A\&A, 411, 257

Schmidt, W., Hofmann, A., Balthasar, H., Tarbell, T. D., \& Frank, Z. A. 1992, A\&A, 264, L27

Shine, R. A., Title, A. M., Tarbell, T. D., et al. 1994, ApJ, 430, 413

Skumanich, A., \& Lites, B. W. 1994, in Solar Magnetic Fields, 200

Solanki, S. K. 2003, A\&AR, 11, 153

Solanki, S. K., \& Montavon, C. A. P. 1993, A\&A, 275, 283

Spruit, H. C. 1981, in The Physics of Sunspots, 359

Spruit, H. C. 2005, in preparation

Stanchfield, D. C. H., Thomas, J. H., \& Lites, B. W. 1997, ApJ, 477, 485

Thomas, J. H. 1988, ApJ, 333, 407

Title, A. M., Frank, Z. A., Shine, R. A., et al. 1992, in NATO ASIC Proc., Sunspots: Theory and Observations, 375, 195

Title, A. M., Frank, Z. A., Shine, R. A., et al. 1993, ApJ, 403, 780

Tritschler, A., Schlichenmaier, R., Bellot Rubio, L. R., et al. 2004, A\&A, 415, 717

van Noort, M., Rouppe van der Voort, L. H. M., \& Löfdahl, M. G. 2005, Sol. Phys., in press

Weiss, N. O., Thomas, J. H., Brummell, N. H., \& Tobias, S. M. 2004, ApJ, 600, 1073

Westendorp Plaza, C., del Toro Iniesta, J. C., Ruiz Cobo, B., et al. 1997, in 1st Advances in Solar Physics Euroconference, Advances in Physics of Sunspots, ASP Conf. Ser., 118, 202

Westendorp Plaza, C., del Toro Iniesta, J. C., Ruiz Cobo, B., et al. 2001, ApJ, 547, 1130

Wiehr, E., \& Stellmacher, G. 1989, A\&A, 225, 528 